\title{
The oxygen sensor Prolyl hydroxylase domain 2 regulates the in vivo suppressive capacity of regulatory $T$ cells
}

\author{
Yousra Ajouaou ${ }^{1,2}$, Abdulkader Azouz ${ }^{1,3}$, Anaëlle Taquin ${ }^{1,2}$, Hind Hussein ${ }^{1,2}$, \\ Fabienne Andris $^{1,2}$, Muriel Moser ${ }^{1,2}$, Stanislas Goriely ${ }^{1,2,3}$ and Oberdan \\ Leo $^{1,2^{*}}$
}

${ }^{1}$ UCR-I (ULB Centre for Research in Immunology), ${ }^{2}$ Laboratoire d'Immunobiologie, ${ }^{3}$ Institute for Medical Immunology, Université Libre de Bruxelles (ULB), Gosselies, Belgium

*Corresponding author: Oberdan Leo (Oberdan.Leo@ulb.be), Laboratoire d'Immunobiologie, IBMM, Rue des Profs Jeener et Brachet 12, 6041 Gosselies (BELGIUM) 


\section{Abstract}

The oxygen sensor PHD2 (prolyl hydroxylase domain 2) plays an important role in cell hypoxia adaptation by regulating the stability of HIF proteins (HIF1 $\alpha$ and HIF2 $\alpha$ ) in numerous cell types including $T$ lymphocytes. The role of oxygen sensor on immune cells, in particular on regulatory $T$ cell (Treg) function, has not been fully elucidated. The purpose of our study was to evaluate the role of PHD2 in the regulation of Treg phenotype and function. We demonstrate herein that selective ablation of PHD2 expression in Treg (PHD2 ${ }^{\Delta \text { Treg }}$ mice) leads to a spontaneous systemic inflammatory syndrome, as evidenced by weight loss, development of a rectal prolapse, splenomegaly, shortening of the colon and elevated expression of IFN-y in the mesenteric lymph nodes, intestine and spleen. PHD2 deficiency in Tregs led to an increased number of activated CD4 conventional T cells expressing an effector/Th1-like phenotype. Concomitantly, the expression of innate-type cytokines such as IL1- $\beta$, IL$12 \mathrm{p} 40, \mathrm{IL}-12 \mathrm{p} 35$ and TNF- $\alpha$ was found to be elevated in peripheral (gut) tissues and spleen. PHD2 ${ }^{\Delta T r e g}$ mice also displayed an enhanced sensitivity to DSS-induced colitis and to toxoplasmosis, suggesting that PHD2-deficient Tregs do not efficiently control inflammatory response in vivo, in particular immune responses characterized by IFN- $\gamma$ production. Further analysis revealed that Treg dysregulation is largely prevented in

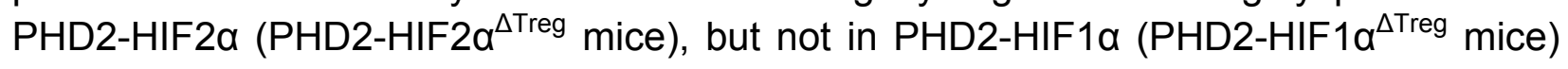
double KOs, suggesting an important and possibly selective role of the PHD2-HIF2 $\alpha$ axis in the control of Treg function. Finally, the transcriptomic analysis of PHD2-deficient Tregs revealed an altered expression of several chemokine receptors including CXCR3, a finding corroborated by the altered in vivo localization of PHD2-deficient Tregs in splenic tissues. Collectively, these findings uncover an important role of the PHD2HIF2 $\alpha$ axis in regulatory T cell positioning and trafficking.

Key words: $\mathrm{CD}^{+} \mathrm{T}$ cell lymphocytes/regulatory T cells/Oxygen sensor/ Hypoxia inducible factor 


\section{Introduction}

$\mathrm{CD}^{+}$regulatory $\mathrm{T}$ cells (Tregs), accounting for approximately $5-10 \%$ of total circulating $\mathrm{CD}^{+} \mathrm{T}$ cells, represent a critical subset of $\mathrm{T}$ lymphocytes involved in immune homeostasis. Through a broad set of effector mechanisms, these cells contribute to immune tolerance to self-constituents and to mucosal antigens derived from the commensal microflora and food ${ }^{1,2}$. Tregs also participate in the resolution of inflammatory responses ${ }^{3}$ and play an important role in maternal immunotolerance against the semi-allogeneic fetus ${ }^{4}$. While critical to maintain tissue integrity, excessive activation of Tregs impedes adequate immune responses to tumors and pathogens, suggesting a tight control of their suppressive activity and tissue localization ${ }^{5-7}$. Although uniformly characterized by the expression of the lineage-specific, Foxp3 transcription factor $^{1}$, regulatory $T$ cells display a wide range of phenotypic and functional properties that allow them to migrate to specific sites and suppress a variety of immune reactions including inflammatory ${ }^{8}$ and humoral responses ${ }^{9}$. Although diverse, the mechanisms whereby Tregs antagonize the activity of immune effectors are largely paracrine in nature $^{10}$. Short-range suppressive mechanisms include competition for nutrients and/or growth factors (mostly cytokines), secretion of immunosuppressive factors and direct, contact-mediated, inactivation of antigen presenting cells ${ }^{11}$. These findings suggest that Tregs have to adapt to multiple lymphoid and non-lymphoid environments and suppress immune responses in a context and tissue-dependent fashion ${ }^{12,13}$.

Oxygen represents an essential component of cellular bioenergetics and biochemistry. Because oxygen tension varies according to tissues and pathophysiological states ${ }^{14}$, cells need to adapt to fluctuations in oxygen availability in order to maintain an adequate functional and metabolic status. Of note, low oxygen availability (hypoxia) plays a critical role in the pathophysiology of many immune disorders ${ }^{15,16}$. Inflammation, in particular, is thought to reduce oxygen availability to tissues by affecting microvascular form and function, and through the recruitment of highly oxygen consuming inflammatory cells producing NADPH oxidase-derived reactive oxygen species ${ }^{17}$.

Immune cells patrolling through lymphoid and non-lymphoid tissues need therefore to readily adapt to varying oxygen concentration levels in order to exert their function ${ }^{18}$, suggesting an important role for oxygen sensors in immune regulation. Several hypoxia-sensitive pathways are known to enable single cell survival in low oxygen settings. In particular, reduced oxygen levels are directly sensed by a family of oxygen-dependent prolyl hydroxylases (PHD or EGLN, comprising three members) ${ }^{19}$, although other mechanisms mediated by oxygen-sensitive histone lysine demethylases (KDM) and the cysteamine dioxygenase/N-degron pathway have been recently uncovered $^{20}$. Hypoxia-inducible factors (HIFs), a set of evolutionary conserved transcriptional regulators, represent the best-described substrates of PHDs. These 
factors are heterodimers composed of a HIFa subunit whose stability is directly controlled by oxygen availability, and a constitutively expressed HIF1 $\beta$ subunit (also known as ARNT) ${ }^{21}$. Following the initial characterization of the first member of HIFa family (HIF1 $\alpha$ ), two additional members, HIF2 $\alpha$ and HIF3 $\alpha$ have been identified and shown to be similarly regulated by $\mathrm{O}_{2}$ availability and to bind to HIF $1 \beta^{22}$. In normoxia, PHDs, whose affinity for oxygen is low, and comparable to atmospheric concentrations, catalyze the prolin-hydroxylation of HIFa subunits ${ }^{23}$. This post-translational modification allows recognition and ubiquitination of HIFa subunits by the E3 ubiquitin ligase Von Hippel-Lindau protein ( $\mathrm{pVHL}$ ) and subsequent degradation by the proteasome ${ }^{24}$. In hypoxia, non-hydroxylated alpha subunits escape degradation, and translocate to the nucleus where they bind to constitutively expressed and stable beta subunits to constitute an active heterodimer able to regulate gene expression. This process promotes transcriptional regulation of numerous genes ultimately leading to increased oxygen supply (such as angiogenesis) and promotion of anaerobic metabolism ${ }^{25}$.

Multiple levels of complexity of this major regulatory axis have been recently uncovered. As previously suggested, the presence of several members of the PHD and HIF families suggests specialized functions of PHD-HIF pairs during ontogeny and in selected tissues ${ }^{26}$. In particular, while HIF1a appears as ubiquitously expressed in all metazoans, HIF2 $\alpha$ represents a late acquisition of vertebrates, displaying a more restricted tissue expression pattern ${ }^{27}$. Although these factors bind to similar sequence motifs (hypoxia response elements or HREs) and regulate the expression of a shared set of genes, both HIF1 $\alpha$ and HIF2 $\alpha$ specific gene targets have been identified in multiple tissues ${ }^{25,28}$. Further complexity in this pathway has been provided by the identification of additional, non-HIF-related, PHD substrates, suggesting a role of proline hydroxylation in other settings requiring oxygen-dependent regulation ${ }^{29}$.

Hypoxia plays a dual role in inflammation and in the regulation of immune responses. In most settings, hypoxia promotes inflammation, while in some instances, such as in tumor sites, low oxygen levels generally cause unresponsiveness of immune effectors, thus favoring tumor growth. The often-opposing effects displayed by HIF activation on the activity of immune cells equally match this complexity ${ }^{30}$. Previous work has indeed highlighted the important role of the PHD-HIF axis in regulating both innate and adaptive immune effectors ${ }^{26}$. Although the analysis of mouse strains in which the expression of members of the HIF or PHD families has been inactivated in immune cell subsets has confirmed the important role for this axis in immune regulation (see references in the Discussion section), the precise role of PHD-HIF signaling in determining immune cell function and fate remains ill-defined. To better understand the mechanism whereby oxygen levels may regulate immune responsiveness, a detailed analysis of the role of members of this family of oxygen sensors on selected immune cell populations is warranted. Using a genetic approach, we demonstrate in this study that 
mice in which expression of PHD2 is selectively inactivated in regulatory T cells display a spontaneous inflammatory syndrome characterized by altered immune homeostasis at the steady-state and high sensitivity to inflammatory diseases. While retaining fully functional suppressive activity in vitro, PHD2-deficient Tregs display a HIF2 $\alpha$-dependent, altered tissue localization and reduced capacity to control spontaneous immune activation. Collectively, our findings highlight an important role for the PHD2-HIF2 $\alpha$ axis in maintaining immune homeostasis, calling for caution in the therapeutic development of pan-PHD inhibitors for treating tissue damage induced by transient hypoxia. 


\section{Results}

\section{Deletion of PHD2 in Tregs leads to a systemic, Type-1-like, inflammatory syndrome associated to altered Treg numbers and phenotype.}

Based on the predominance of PHD2 expression in Tregs over other members of the prolyl hydroxylases family (Fig. S1), we generated a mouse strain lacking PHD2 expression in Tregs (identified as $P H D 2^{\Delta T r e g}$ ) as described in the Methods section. These mice displayed a strongly reduced expression of PHD2 mRNA in Tregs, while retaining control level expression of this enzyme in other, non-Treg spleen and peripheral lymph node cells (Fig. S1). Spontaneous expression of GLUT1, a well-known target of HIF1 $\alpha$, was also only found in Foxp3-expressing cells in these mice, further supporting the selective depletion of PHD2 in Tregs (Fig S1). While fertile and viable, over $70 \%$ of these mice developed a spontaneous inflammatory syndrome, characterized by weight loss, episodes of anal prolapse, splenomegaly, hemorrhagic abdomen and reduced colon length (Fig. 1a-e). Although the frequency of $\mathrm{CD}^{+}$and $\mathrm{CD}^{+}$conventional $\mathrm{T}$ lymphocytes in several lymphoid organs were not altered in $\mathrm{PHD}^{\Delta \mathrm{Treg}}$ mice (Fig. 1f), these Foxp3-negative cells displayed signs of spontaneous activation, as evidenced by the significant increase in cells expressing an effector and memory-like phenotype. (Fig. 1g, h). Confirming these findings, intracellular staining of short-term stimulated T cells (using pharmacological agents bypassing TCR signaling) revealed an increased capacity of conventional T cells from PHD2 ${ }^{\Delta T r e g}$ mice to produce IFN- $y$, while retaining control-like production of IL-17 (Fig. 1i). The ex-vivo evaluation of mRNA abundance in whole, unfractionated, $\mathrm{mLNs}$ similarly showed a significantly elevated expression of Type 1-associated adaptive and innate cytokines including IFN- $\gamma$, IL-1 $\beta$, both IL-12 subunits and TNFa (Fig. 1j). Overall, these observations point to the establishment of a Th1-like, pro-inflammatory environment in mice possessing PHD2deficient Tregs. Much to our surprise, flow cytometric analysis of lymphoid organs from naive animals revealed an increased frequency of Treg cells in the spleen, lymph nodes and lamina propria of PHD2 ${ }^{\triangle T r e g}$ mice, when compared to control animals (Fig. 2a). No difference in the relative frequency of Treg subsets identified by the co-expression of Foxp3 with either naive and memory markers (Fig. 2b) or with master transcription factors T-bet, GATA3 or RORyt (Fig. 2c) was noted in these mice. The phenotype of PHD2-deficient Tregs was however significantly altered, showing a slight, but statistically significant reduction in the expression of Foxp3 (Fig. 2d), accompanied by reduced expression of the CD25, ICOS, and CD44 markers and enhanced expression of PD-1 (Fig. 2e). Of note, neither CTLA4 (Fig. 2e) nor IL-10 (Fig. 2f) expression was altered in PHD2-deficient Tregs. To evaluate the functional consequences of PHD2-deletion on Treg development, we generated Tregs from naive, conventional T cells using a wellestablished in vitro protocol. In keeping with in vivo observations, culture of $\mathrm{CD} 4^{+} \mathrm{T}$ conv from $\mathrm{PHD} 2^{\Delta T r e g}$ mice led to a consistently higher yield of Foxp3-expressing cells when 
activated in the presence of Treg-inducing cytokines (Fig. $\mathbf{2 g}, \mathbf{h}$ ). In contrast to their in vivo counterparts however, these induced Tregs displayed control level expression of Foxp3 (Fig. 2i).

\section{In vivo reduced suppressive function of PHD2-deficient Tregs.}

To evaluate the suppressive capacity of PHD2-deficient Tregs cells, ex-vivo purified CD45.2 expressing Tregs from control and $\mathrm{PHD} 2^{\Delta T r e g}$ mice were adoptively cotransferred into syngeneic Rag-deficient mice with CFSE-labelled, CD45.1-expressing $\mathrm{CD}^{+}$naive $\mathrm{T}$ lymphocytes (Fig. 3a). In the absence of Tregs, transferred naive cells rapidly divided and acquired an effector-like phenotype, a well-established consequence of homeostatic proliferation in a lymphopenic environment (Fig. 3b). Addition of WT Tregs in the inoculum led to a significant reduction of conventional $\mathrm{T}$ cell proliferation and phenotype switch, while PHD2-deficient Tregs appeared functionally impaired in this assay (Fig. 3b-d). Lack of suppressive activity of these Tregs was not a consequence of reduced viability and/or in vivo survival, as shown by the normal recovery rate of both Treg-populations at the time of assay read-out (Fig. 3e). In contrast, when tested in vitro, PHD2-deficient Tregs consistently displayed a fully functional suppressive activity (Fig. 3f, g).

\section{Increased susceptibility of PHD2 ${ }^{\Delta \text { Treg }}$ mice to Type-1 experimental inflammation}

A series of experimental acute and chronic inflammatory models were employed to further evaluate the capacity of $\mathrm{PHD}^{\triangle \mathrm{Treg}}$ mice to sustain an in vivo inflammatory challenge. We first exposed mice to a chemical-induced colitis protocol. This assay revealed an increased sensitivity of $\mathrm{PHD}^{\triangle \mathrm{Treg}}$ mice to most DSS-induced inflammatory manifestations, including weight loss (Fig. 4a), survival (Fig. 4b), clinical score (Fig. 4c) and colon length (Fig. 4d). No difference was noted however in crypt morphology induced by DSS in both mouse strains (Fig. 4e). Similar observations were made when mice were acutely infected with Toxoplasma gondii, a model of infection-induced pathology (Fig. 4f). Infected PHD2 ${ }^{\Delta T r e g}$ mice displayed increased weight loss (Fig. 4g), reduced colon length (Fig. $\mathbf{4 h}$ ) and increased frequency of activated cells characterized by an effector-like phenotype (Fig. 4i) and IFN-Y secretion capacity (Fig. 4j). Infected $\mathrm{PHD}^{\Delta{ }^{\Delta r e g}}$ mice also displayed a decrease in Treg T-bet $^{+}$frequency, a population known to control Th1 inflammation during toxoplasmosis ${ }^{31}$ (Fig. 4k). Overall, PHD2 ${ }^{\Delta T r e g}$ mice displayed an uncontrolled expansion of Th1-like cells following experimental toxoplasmosis. In contrast, both PHD2-deficient and sufficient mouse strains were equally sensitive to enteritis induced upon injection of anti-CD3 antibodies (Fig. S2), a model known to induce the predominant expansion of Th17-like, inflammatory effectors in vivo ${ }^{32}$. The role of uncontrolled IFN-y secretion in mediating the pro-inflammatory status of this mouse strain was further confirmed by the observation that ubiquitous loss of IFN-y gene expression largely reversed the phenotypical and cellular altered status of PHD2 $^{\Delta \text { Treg }}$ mice (Fig. S3). 
Concomitant loss of HIF2 $\alpha$, but not HIF1a expression, partially corrects the proinflammatory phenotype of PHD2 ${ }^{\Delta \text { Treg }}$ mice.

Based on the notion that HIF1 $\alpha$ and HIF2 $\alpha$ represent well-described targets of PHD2, we established a series of conditional KOs mouse strains in an attempt to identify the molecular pathway responsible for the decreased functional activity of PHD2-deficient Tregs at steady state (see Fig. S4 for strain validation). Treg-selective deletion of HIF1 $\alpha$ and HIF2 $\alpha$ expression alone did not significantly alter colon length (used as a proxy for spontaneous inflammation) nor general T cell immune homeostasis (Fig. S4). The same observation was made for double HIF1 $\alpha$ and HIF2 $\alpha$ KOs (data not shown). In marked contrast, combined deletion of PHD2 and HIF2 $\alpha$ reversed some the inflammatory symptoms observed in PHD2 ${ }^{\Delta T r e g}$ mice, such as splenomegaly and colon length (Fig. 5a, b). Treg specific, PHD2-HIF1a double KOs were virtually indistinguishable from single PHD2 KO according to these morphological criteria. Noteworthy however, Tregspecific PHD2-HIF1a double KOs mice were born at sub-mendelian ratios, and displayed a marked weight loss during adult life and reduced viability, indicative of a more pronounced pro-inflammatory status (data not shown). This mouse strain also displayed a tendency toward increased expansion of Th1-like cells in peripheral lymph nodes (Fig. 5e). PHD2-HIF1 $\alpha-H I F 2 \alpha$ triple KOs and PHD2-HIF2 $\alpha$ double KOs displayed a similar phenotype, establishing a predominant role for HIF $2 \alpha$ over HIF1 $\alpha$ in mediating the effects of PHD2 on the capacity of Treg to regulate immune homeostasis at rest. Similarly, lack of HIF2 $\alpha$ expression largely reversed the altered phenotype of conventional T cells induced by loss of Treg-associated PHD2 expression. Indeed, cells from double (PHD2-HIF2 $\alpha$ ) and triple (PHD2-HIF1 $\alpha-H I F 2 \alpha)$ Treg specific KOs displayed a near normal phenotype (based on CD62L and CD44 expression) and propensity to secrete IFN-y (Fig. 5c-e). Finally, loss of Treg-associated expression of HIF2a also reversed the expansion of Treg numbers (Fig. 5f) and restored Foxp3 protein expression to near-control levels (Fig. $\mathbf{5 g}$ ).

Transcriptomic analysis identifies cell survival, response to chemokines and STAT1-mediated signaling as target pathways of the PHD2-HIF2 $\alpha$ axis in Tregs. Collectively, the previous observations suggest that the PHD2-HIF2 $\alpha$ regulatory axis confers to Tregs the capacity to control the spontaneous, type-1 like, activity of conventional $\mathrm{T}$ cells. To identify PHD2-dependent signaling pathways operating in Tregs, splenic Foxp3-expressing cells were purified from all mouse strains described in this manuscript and their transcriptome analyzed following bulk RNA-seq. A set of 532 genes were found differentially expressed between WT and PHD2-deficient Tregs (a summary-list of upregulated and downregulated pathways in PHD2 ${ }^{\Delta T r e g}$ mice vs WT mice is provided in Fig. S5). Differential gene expression analysis between all mouse strains studied, identifies 1868 genes differentially expressed between groups. An unsupervised clustering of the differentially expressed genes led to the identification of 20 clusters, as shown in Fig. 6a. To identify gene clusters that were specifically involved 
in the immune homeostatic control of naive mice, the RNA-seq data were filtered and grouped by k-mean clustering. We next searched for sets of genes whose expression best correlated with an arbitrary inflammatory index, established based on previously described findings (mostly colon length, splenomegaly and spontaneous conventional T cell activation status) and summarized in Fig. 6b. In particular, while concomitant deletion of HIF1a expression worsened the inflammatory status of PHD2 ${ }^{\Delta \text { Treg }}$ mice, loss of HIF2 $\alpha$ expression mitigated most inflammatory-related parameters at rest. We therefore clustered genes according to a "gradient of disease severity" and grouped them in sets of gene whose expression decreased (cluster 10, Fig. 6c) or increased (cluster 11, Fig. 6d) accordingly. Gene ontology analysis of these clustered gene sets revealed the following. Reduced expression of cell death-related and gain of survivalassociated gene expression correlated with the increased frequency of Tregs in the corresponding mouse strains (Fig. 6c, d). Not surprisingly, the expression of genes associated with anti-inflammatory responses was gradually lost according to the same severity gradient. Finally, genes, associated with $\mathrm{T}$ cell migration, including several chemokine receptors, also displayed an ordered loss of expression along the same gradient (Fig. 6c). For comparison purposes, genes whose expression is restored to control levels upon combined deletion of PHD2 and HIF1a were also examined. As expected from previously published findings, these HIF1a-dependent biological pathways included glycolysis and angiogenesis (Fig. 6e). Ingenuity Pathway Analysis was performed in order to identify possible upstream regulators affecting expression of downstream genes identified in clusters 10 and 11. This analysis led to the identification of STAT1 as a putative upstream transcription factor regulating the expression of a set of genes whose expression was altered in PHD2-deficient Tregs (Fig. 7a). Since STAT1 mRNA expression was not altered by PHD2 invalidation (as revealed by RNA-Seq analysis), we tested the capacity of STAT1 to undergo phosphorylation in response to IFN-y. This set of experiments led to the identification of a defective, accumulation of phopho-STAT1 in PHD2-deficient Tregs (Fig. 7b, c), while the levels of total STAT1 protein appeared unaffected (Fig. 7d). Noteworthy, concomitant deletion of HIF2 $\alpha$ restored a near control response to IFN-y in PHD2 deficient Tregs (Fig. 7b-d). In keeping with the observed pro-inflammatory phenotype associated with these mouse strains, conventional $\mathrm{T}$ cells from $\mathrm{PHD} 2^{\Delta \mathrm{Treg}}$ mice displayed an augmented response to IFN-Y (as judged by pSTAT1 accumulation), partially reversed in mice bearing Tregs lacking both PHD2 and HIF2 $\alpha$ expression (Fig. 7b, left pannel). Overall, these observations indicate that PHD2 expression controls the response of Tregs to IFN-Y.

\section{The PHD2-HIF2 $\alpha$ axis regulates adequate positioning of Tregs in the splenic white pulp.}

STAT1 is known to regulate CXCR3 expression, a chemokine receptor known to confer to Tregs the capacity to adequately control Th1-like inflammatory responses in vivo ${ }^{33-35}$. Accordingly, and in keeping with the transcriptomic data, splenic and mesenteric lymph 
node PHD2-deficient Tregs expressed reduced levels of CXCR3 (Fig. 8a). CXCR3 expression was largely restored to control levels in PHD2-HIF2 $\alpha$ double KOs (Fig. 8a). Because PHD2-deficient Tregs display an adequate suppressive activity in vitro, we postulated that these cells might be inadequately positioned in vivo to fully exert their suppressive function. The relative location of regulatory (identified as $\mathrm{CD}^{+} \mathrm{YFP}^{+}$cells) and conventional (marked as CD4 ${ }^{+} \mathrm{YFP}^{-}$) lymphocytes within the splenic tissues of naive mice from the indicated strains was analyzed by immunochemistry and image analysis. Tregs from PHD2 ${ }^{\Delta \text { Treg }}$ mice were predominantly found in the splenic red pulp, while WT Tregs appeared to preferentially localize within the lymphocyte-rich white pulp (Fig. 8b, c). Moreover, PHD2-deficient Tregs appeared as mostly confined to the periphery of the white pulp, rather than homogenously distributed in the T cell-enriched zone (Fig 8b, d). Finally, and although the biological significance of this observation remains to be established, PHD2-deficient Tregs were found at higher frequencies in "Treg-only clusters", possibly suggesting that they were less inclined to establish close contacts with conventional T cells (Fig. 8e). Altered Treg positioning of PHD2-deficient cells was largely restored by the concomitant loss of HIF2a expression. This observation is compatible with transcriptomic analysis revealing altered expression of several chemokine receptors and suggests an important role of the PHD2-HIF2 $\alpha$ axis in regulatory $\mathrm{T}$ cell migration and trafficking. 


\section{Discussion}

The present study highlights the important role of the prolyl-hydroxylase PHD2 in the regulation of Treg function. PHD2-deficient Tregs were found in increased numbers in vivo at steady state (Fig. 2a), albeit with an altered phenotype. In particular, the expression of molecules known to be associated with optimal suppressive activity (such as Foxp3, ICOS and CD25) $)^{1,36,37}$ was marginally decreased, while expression of PD-1, a marker associated with altered functional activity of many immune cells including Tregs $^{38,39}$ was augmented. Of note, other molecules known to play an important role in Treg function appeared as expressed at optimal levels (cf CLTA4 and IL-10). Mice selectively lacking PHD2-expression in Treg displayed a proinflammatory phenotype (with early manifestations of gastrointestinal tract inflammation), associated to an altered homeostatic profile of splenic conventional $T$ cells. Several observations strongly indicate that these phenotypic alterations were due to the specific impairment in Treg function consequent to the loss of PHD2 activity. Despite previous reports describing the stochastic activity of the Foxp3-Cre-YFP allele in non-Tregs leading to the recombination of some, but not all alleles ${ }^{40}$, we consistently found a control-level expression of PHD2 in all conventional T cell subset tested (Fig. S1). Secondly, co-transfer of highly purified Tregs with WT naive $\mathrm{CD}^{+}$conventional target cells clearly demonstrated an intrinsic role of PHD2 in regulating Treg function. Finally, and this will be discussed further below, mice bearing double PHD2-HIF2 $\alpha$-deficient Tregs recover a near-control phenotype, further excluding a major influence of the genetic background on the observed phenotype.

Our observations confirm and further extends observations from a study published during completion of our work ${ }^{41}$, indicating a specific, non-redundant role for PHD2 in controlling Treg activity in vivo. Although we have not specifically addressed the role of PHD2 in thymic vs peripherally induced Tregs, it is noteworthy that lack of PHD2 expression did not affect the generation of iTreg in vitro, suggesting a normal response of these cells to Treg-inducing cytokines such as IL-2 and TGF $\beta$.

Based on the notion that hypoxia-induced factors represent major substrates of PHD2, we generated a series of mouse strains to evaluate the relative role of HIF1 $\alpha$ and HIF2 $\alpha$ in regulating Treg phenotype and function. The combined loss of PHD2 and HIF2 $\alpha$ but not HIF1 $\alpha$, corrected some, but not all abnormalities found in the PHD2 ${ }^{\Delta \text { Treg }}$ mouse strain.

To uncover the mechanism whereby the PHD2-HIF2 $\alpha$ axis regulates the capacity of Tregs to exert a homeostatic control over conventional T cells, a large transcriptomic analysis was undertaken. To be able to isolate genes specifically involved in the control of Treg activity in naive animals, we took advantage of the graded pro-inflammatory status of the mouse strain generated (such as colon length and spontaneous activation of Tconv cells, see Fig. 5) to identify gene clusters whose expression correlated with 
Treg-mediated immune homeostasis. This analysis led us to identify important pathways providing mechanistic insights into the role of the PHD2-HIF2 $\alpha$ axis in Treg biology. In particular, loss of PHD2 led to altered expression of genes coding for chemokine receptors and adhesion molecules, suggesting a potential role of this oxygen sensor in chemotaxis and traffic. This conclusion is of particular interest in light of two observations described in this study. As previously discussed, PHD2 ${ }^{\Delta T r e g}$ mice displayed a selective expansion of Th1-prone effectors in all lymphoid organs examined (Fig. 1). Accordingly, ubiquitous loss of IFN-Y expression strongly attenuated the proinflammatory phenotype of mice with PHD2-deficient Tregs (Fig S3), thus suggesting a specific role for PHD2 in endowing Tregs to control Th1-like immune responses in vivo. Based on well-described role for Treg-expressed CXCR3 in modulating Th1-like responses in vivo ${ }^{42,43}$, and the reduced expression of this chemokine receptor described in the present study (Fig. 6c and Fig. 8a), it is tempting to speculate that the reduced capacity of PHD2-deficient Tregs to control Th1 responses is a consequence of altered CXCR3 expression. Although indirect, this hypothesis is supported by the analysis of Treg positioning within the spleen, shown in Fig. 8. PHD2-deficient Tregs appeared as largely misplaced when compared to their WT counterpart, with limited recruitment in T cell-rich areas of the spleen. In agreement with our working hypothesis, response to CXCR3 ligands has been recently shown to determine the precise positioning of effector and memory CD8 cells in peripheral lymph nodes ${ }^{44}$. Further studies would be required to identify the precise mechanism at work, since the expression of many potential chemokine receptors (including CXCR4, known to exert inhibitory function over other chemokine receptors ${ }^{45}$ ) and adhesion molecules (such as Ly6a or CD44) appear under the control of the PHD2-HIF2 $\alpha$ axis in Tregs. Of note, the lack of pro-inflammatory phenotype in animals lacking PHD2 in all $\mathrm{T}$ cells subsets ${ }^{46}$ may be explained by a conserved Treg-Tconv co-localization in these mice. This observation may also provide a plausible explanation for the capacity of PHD2-deficient Treg to retain a normal suppressive function in vitro, but not in vivo. Finally, the IPA analysis conducted on the transcriptomic data led to the identification of STAT1 as a potential common regulator of many genes whose expression was under the control of the PHD2-HIF2 $\alpha$ axis, including in particular $\mathrm{CXCR}^{34}$.

Collectively, the observations reported in this study demonstrate a non-redundant role for PHD2 in controlling survival, phenotype, migration properties and the capacity of Tregs to control Th1-like responses. These biological responses appear under the control of HIF2 $\alpha$, and largely independent of HIF1a-regulated metabolic pathways. Although the role of the PHD2-HIF2 $\alpha$ axis has been previously highlighted by Yamamoto and colleagues using an alternative, shRNA-based approach ${ }^{41}$, our observations do not fully concur with the previous study on two grounds. In contrast to PHD2 knock-down (PHD2-KD) cells, PHD2-genetically deficient Tregs (PHD2-KO, this study) retained full suppressive capacities in vitro. Furthermore, no signs of reversal to an effector state was 
found in PHD2-KO regulatory T cells, whereas downregulation of PHD2 expression led to an increased expression of T-bet, GATA-3 and TNFa. Notably, PHD2-KD Tregs were able to induce skin-graft rejection in the absence of bona-fide effector cells, suggesting a possible acquisition of effector function by these cells. Although these observations are compatible with a possible gene-dosage effect of PHD2 on Treg biology, further studies are needed to identify the mechanism at work in these two experimental models.

In any events, both studies concur in identifying a possible deleterious role of HIF2 $\alpha$ overactivation in the control of regulatory T cell function. These findings appear at odds with a recent publication by Tzu-Sheng $\mathrm{Hsu}$ and colleagues in which deletion of HIF2 $\alpha$, but not HIF1a, expression was found to negatively affect Treg function ${ }^{47}$. Of note, concomitant deletion of both HIF1 $\alpha$ and HIF2 $\alpha$ restored the suppressive activity of Tregs ${ }^{47}$. An elegant hypothesis, put forward by these authors, may help reconcile some of these apparently contradictory observations.

Most experimental evidence concurs with a dual role of HIF1a in Treg differentiation and stability. In setting of sub-optimal Treg-inducing conditions, HIF1a may promote adequate expression of Foxp3 by differentiating Tregs. Once the Treg phenotype has been fully acquired, HIF1a protein expression is reduced following interaction with Foxp $3^{48}$, thus explaining the relative lack of influence of HIF $1 \alpha$ on differentiated Tregs. As a consequence, HIF1a-KO Tregs retain full suppressive activity $^{47}$. The interaction between HIF1 $\alpha$ and Foxp3 can however also lead to Foxp3 protein degradation, and thus Treg instability. As a consequence, forced stabilization of HIF1a (such as observed in triple PHD KOs ${ }^{46}$ or pVHL-deficient Tregs ${ }^{49}$ ) leads to loss of Foxp3 expression and Treg identity and acquisition of pro-inflammatory functions. Inflammation observed in these mouse strains can be largely attributed to the proinflammatory influence of ex-Tregs. As discussed for HIF1 $1 \alpha$, HIF2 $\alpha$ also appears to regulate Treg stability, albeit in a different direction. Despite a normal phenotype at rest, mice displaying HIF2 $\alpha$-deficient Tregs are largely defective in suppressing inflammation in the gut and in the lungs ${ }^{47}$. This pro-inflammatory phenotype was largely explained by the HIF1a-dependent reprogramming of HIF2a-deficient Tregs into IL-17 secreting cells. Collectively, the available literature points to a central role for HIF1 $\alpha$ in determining Treg stability and function in vivo. Depending on the biological pathway leading to its increased expression and/or protein stabilization, HIF1a promotes the differentiation of Tregs into IFN-Y (in triple PHD KOs or pVHL-deficient Tregs) or IL-17 (in HIF-2adeficient Tregs) secreting cells. Although the mechanism underlying the acquisition of Th1 vs Th17-like profiles in these models remains to be established, the induction of a glycolytic metabolism is probably instrumental in mediating Treg instability ${ }^{50}$.

In the present study, loss of HIF1a expression did not revert the phenotype of PHD2-HIF2 $\alpha$-deficient Tregs, despite reestablishing a control-like expression of pro- 
glycolytic genes (Fig. 6e). Accordingly, PHD2-deficient Tregs did not acquire the capacity to produce pro-inflammatory cytokines (Fig. S1), nor displayed any significant loss of Foxp3 expression upon in vitro culture (Fig. 2i) or in vivo transfer (Fig. 3e). Thus, the available evidence suggests that in PHD2-sufficient cells HIF2 $\alpha$ allows adequate Treg function by negating the influence of HIF1a on Foxp3-expression, while overactivation of HIF2 $\alpha$ activity secondary to the loss of PHD2 expression leads to altered Tregs phenotype, most probably via a STAT1-dependent pathway.

Considering the specific role of PHD2, it is worth mentioning that both the transcriptomic data and our own unpublished observations (indicating an increased sensitivity of triple PHD2-HIF1 $\alpha-H I F 2 \alpha$ Tregs specific KO mice to chemical induced colitis) suggest that while the capacity of Tregs to control tissue homeostasis in the naive state is under the predominant control of the PHD2-HIF2 $\alpha$ axis, other, non-HIFs PHD2-substrates probably play an important role in Treg biology under strong inflammatory settings. Finally, the present study suggest that some caution should be exerted in the administration of PHD inhibitors presently considered for the treatment of renal anemia ${ }^{51}$, inflammatory bowel diseases ${ }^{52}$ as well as Parkinson's disease ${ }^{53}$, as these compounds may display some pro-inflammatory effects via the alteration of Treg migration and function in vivo. 


\section{Methods}

Mice. C57BL/6 mice were purchased from Envigo (Horst, The Netherlands). PHD2 ${ }^{\mathrm{f} / \mathrm{f}}$ mice were provided by P. Carmeliet (VIB-KULeuven, Leuven, Belgium); Foxp3-Cre-YFP mice, developed by $A$. Rudensky ${ }^{54}$ were kindly provided by A. Liston (KU Leuven, Leuven, Belgium); Hif1atm3Rsjo/J $\left(\mathrm{HIF}^{\mathrm{fff}} \mathrm{f}^{\mathrm{f}}\right)$ mice were kindly provided by F. Bureau (Liege University, Liege, Belgium); Epas1tm1Mcs/J $\left(\mathrm{HIF}^{\mathrm{f}} \mathrm{a}^{\mathrm{f} / \mathrm{f}}\right)$ mice were provided by J.A. Lopez (Madrid University, Madrid, Spain); IFNy ${ }^{-/}$, CD45.1 (B6.SJL-Ptprc ${ }^{a}$ Pepc $^{b} / B^{b}{ }^{J}$ ) and RAG2 ${ }^{-/}$mice were obtained from The Jackson Laboratory (Bar Harbor, ME, USA). All mice were backcrossed for more than 10 generations into a C57BL/6 background and housed in individually ventilated cages. Foxp3-Cre-YFP mice were crossed with $\mathrm{PHD}^{\mathrm{f} / \mathrm{f}}, \mathrm{HIF} 1 \mathrm{a}^{\mathrm{f} / \mathrm{f}}, \mathrm{HIF} 2 \mathrm{a}^{\mathrm{f} / \mathrm{f}}$ to produce mice with Treg-specific deletion of PHD2, HIF1a, HIF2 $\alpha$, PHD2-HIF1 $\alpha$, PHD2-HIF2 $\alpha$ or PHD2-HIF1 $\alpha-H I F 2 \alpha$. All mice were used between 12 and 14 weeks of age except for EAE induction, in which mice were used between 8 and 10 weeks. PHD2-sufficient mice (expressing Foxp3-Cre-YFP, or floxed forms of PHD2, HIF1 $\alpha$ and HIF2 $\alpha$-encoding alleles and generated as littermates in our colony) were used as appropriate controls in the early stages of this work. These mice displayed a phenotype indistinguishable from WT mice and were therefore considered as a single experimental group throughout this study in order to reach statistical significance in all experiments. The experiments were carried out in compliance with the relevant laws and institutional guidelines and were approved by the Universite Libre de Bruxelles Institutional Animal Care and Use Committee (protocol number CEBEA-4).

Antibodies, intracellular staining and flow cytometry. The following monoclonal antibodies were purchased from eBioscience: CD278 (ICOS)-biotin, CD27-PeCy7, Foxp3-FITC, RORyt-PE, T-bet-PE; or from BD Biosciences: PD1-PECF594, CXCR3APC CD25-BB515, CD44-PECy7, CD4-A700, CD8-A700, CD4-PB, CD62L-A700, GATA3-PE, RORyt-PECF594, STAT1 (pY701)-A488, IFNy-PE, IL-10-APC, IL-17PerCy5.5, streptavidin-PECy7. Live/dead fixable near-IR stain (ThermoFisher) was used to exclude dead cells. For transcription factor staining, cells were stained for surface markers, followed by fixation and permeabilization before nuclear factor staining according to the manufacturer's protocol (Foxp3 staining buffer set from eBioscience). For cytokine staining, cells were stimulated in media containing phorbol 12-myristate 13acetate $(50 \mathrm{ng} / \mathrm{mL}$, Sigma-Aldrich), ionomycin $(250 \mathrm{ng} / \mathrm{mL}$, Sigma-Aldrich) and brefeldin-A $(1 / 100$, eBioscience) for $3 \mathrm{~h}$. After stimulation, cells were stained for surface markers, followed by fixation and permeabilization before intracellular staining according to the manufacturer's protocol (cytokine staining buffer set from BD Biosciences). For phosphorylation staining, cells were stimulated with IFN-y (50 ng/mL, Peprotech) for 30 min, fixed with formaldehyde and permeabilized with methanol before staining. Flow cytometric analysis was performed on a Canto II (BD Biosciences) and analyzed using FlowJo software (Tree Star). 
T cell cultures. After removal of Peyer's patches and mesenteric fat, intestinal tissues were washed in HBSS 3\% FCS and PBS, cut in small sections and incubated in HBSS $3 \%$ FCS containing 2,5mM EDTA and $72,5 \mu \mathrm{g} / \mathrm{mL}$ DTT for $30 \mathrm{~min}$ at $37^{\circ} \mathrm{C}$ with agitation to remove epithelial cells, and then minced and dissociated in RPMI containing liberase $\left(20 \mu \mathrm{g} / \mathrm{ml}\right.$, Roche) and DNase $\left(400 \mu \mathrm{g} / \mathrm{ml}\right.$, Roche) at $37^{\circ} \mathrm{C}$ for $30 \mathrm{~min}$. Leukocytes were collected after a 30\% Percoll gradient (GE Healthcare). Lymph nodes and spleens were mechanically disrupted in culture medium. $\mathrm{CD}^{+} \mathrm{T}$ cells were positively selected from organ cell suspensions by magnetic-activated cell sorting using CD4 beads (MACS, Miltenyi) according to the manufacturer's protocol, and purified as CD4 ${ }^{+}$ $\mathrm{CD} 44^{\mathrm{lo}} \mathrm{CD} 62 \mathrm{~L}^{\mathrm{hi}} \mathrm{CD} 25^{-}$or $\mathrm{CD}^{+} \mathrm{CD}^{\mathrm{lo}} \mathrm{CD}^{\mathrm{C}} \mathrm{L}^{\text {hi }} \mathrm{YFP}^{-}$by fluorescence activated cell sorting. $\mathrm{T}$ cells were cultured at $37^{\circ} \mathrm{C}$ in RPMI supplemented with $5 \%$ heat-inactivated FBS (Sigma-Aldrich), 1\% non-essential amino acids (Invitrogen), $1 \mathrm{mM}$ sodium pyruvate (Invitrogen), $2 \mathrm{mM}$ L-glutamin (Invitrogen), $500 \mathrm{U} / \mathrm{mL}$ penicillin/500 $\mathrm{mg} / \mathrm{ml}$ streptomycin (Invitrogen), and $50 \mu \mathrm{M} \beta$-mercaptoethanol (Sigma-Aldrich). To generate iTreg cells, cells were cultured in 24 well plates coated with $5 \mu \mathrm{g} / \mathrm{mL}$ anti-CD3 (BioXcell, clone 145$2 \mathrm{C} 11)$ at $37^{\circ} \mathrm{C}$ for $72 \mathrm{~h}$. The culture was supplemented with anti-CD28 $(1 \mu \mathrm{g} / \mathrm{mL}$, BioXcell, clone 37.51), TGF- $\beta$ (3 ng/ml, eBioscience) and IL-2 (10 ng/mL, Peprotech) for optimal iTreg cell polarization.

Treg cell suppression assays. In vitro assay: $\mathrm{CD}^{+} \mathrm{CD}^{\mathrm{lo}} \mathrm{CD}^{\mathrm{l}} 2 \mathrm{~L}^{\mathrm{hi}} \mathrm{CD} 25^{-}$naive $\mathrm{T}$ cells were isolated from the spleen of $\mathrm{CD} 45.1^{+}$mice by cell sorting after positive enrichment for $\mathrm{CD}^{+}$cells using MACS LS columns (Miltenyi) and labelled with carboxyfluorescein diacetate succinimidyl ester (CFSE, ThermoFisher). CD4 ${ }^{+} \mathrm{YFP}^{+} \mathrm{Treg}$ cells were isolated from the spleen of Foxp3-Cre-YFP or PHD2 ${ }^{\Delta T r e g}$ mice by cell sorting. Splenocytes from wild-type B6 mice were depleted in T cells (anti-CD90.2 beads, MACS, Miltenyi) using MACS LS columns (Miltenyi) and used as feeder cells. $4 \times 10^{4}$ CFSE-labelled naive $T$ cells were cultured for $72 \mathrm{~h}$ with feeder cells $\left(1 \times 10^{5}\right)$ and soluble anti-CD3 $(0,5 \mu \mathrm{g} / \mathrm{mL})$ in the presence or absence of various numbers of Treg cells as indicated.

In vivo assay: Rag2 ${ }^{--}$mice were injected i.v with a mixture of naive, CFSE labeled, CD4 ${ }^{+}$ T cells (CD45.1 $\left.1^{+} \mathrm{CD}^{+} \mathrm{CD}^{\mathrm{lo}} \mathrm{CD}^{\mathrm{CL}} \mathrm{L}^{\mathrm{hi}} \mathrm{CD}^{-} 5^{-}\right)\left(1 \times 10^{6}\right)$ and splenic Treg from Foxp3Cre-YFP or PHD2 ${ }^{\Delta \text { Treg }}$ mice $\left(3.3 \times 10^{5}\right)$. Six days after the injection, Rag2 ${ }^{-/-}$mice were sacrificed and $\mathrm{CD}^{+} \mathrm{T}$ cells proliferation and activation analyzed by flow cytometry.

DSS-induced colitis. Foxp3-Cre-YFP or PHD2 ${ }^{\triangle T r e g}$ mice were provided with $2 \%$ DSS (MP Biomedical, 160110) in tap water for five days. On day 5, the DSS-containing water was replaced with normal drinking water and mice were followed during 14 days for body weight, survival, and colitis severity. Colitis severity score was assessed by examining weight loss, feces consistency and hematochezia (Hemoccult SENSA, Mckesson Medical-Surgical, 625078) as described in ref ${ }^{55}$. Colon samples were washed with PBS and rolled from the distal to proximal end, transected with a needle 
and secured by bending the end of the needle and fixed in fresh $4 \%$ paraformaldehyde (Sigma-Aldrich) overnight and further subjected to routine histological procedures for embedment in paraffin and hematoxylin and eosin (H\&E) staining. Tissues were analyzed and scored in a blinded fashion by an independent histopathologist and representative images were subsequently chosen to illustrate key histological findings.

Toxoplasma infection. ME-49 type II Toxoplasma gondii was kindly provided by Dr De Craeye (Scientific Institute of Public Health, Belgium) and was used for the production of tissue cysts in C57BL/6 mice previously (1-3 month) inoculated with three cysts by gavage. Animals were killed, and the brains were removed. Tissue cysts were counted and mice were infected by intragastric gavage with 10 cysts. Mice were sacrificed at day 8 after infection.

Anti-CD3 mAb-induced enteritis. Mice were injected i.p. with a CD3-specific antibody (clone 145-2C11, BioXcell $20 \mu \mathrm{g} /$ mouse) on days 0 and 2 and weighted daily. Mice were sacrificed on day 3 and cytokine production evaluated by qPCR as indicated in the figure legend.

Immunohistochemistry. Freshly removed spleens were embedded in frozen section compound (Leica 3801480) and snap frozen in methylbutane cooled in liquid nitrogen. Cryosections $(16 \mu \mathrm{m})$ were mounted on microscope slides (Superfrost Plus, Thermofischer J1800AMNZ) and washed with PBS. Tissues were fixed in $2 \%$ paraformaldehyde for $5 \mathrm{~min}$ at RT followed by methanol on ice. After 3 washes in PBS, tissues were blocked for 2 hours at RT with a blocking solution containing $2 \%$ Goat serum (Invitrogen 10000C), 2\% BSA (Sigma-Aldrich), 0.1M glycine (Sigma-Aldrich) and $0.01 \%$ Triton-X100 in PBS. The slides were then incubated with the primary antibodies anti-CD4 (Thermofischer 14-9766, used at 1:200) and anti-GFP (Abcam ab13970, used at 1:500) in blocking buffer $\mathrm{O} / \mathrm{N}$ at $4^{\circ} \mathrm{C}$ and then at $\mathrm{RT}$ for 2 hours. After several washes, the secondary antibodies directed against rat IgG (Thermofischer A-11007, used at $1: 1000$ ) and chicken IgY (Thermofischer A-11039, used at 1:1000) were added for 90 min at RT. The slides were then extensively washed with blocking buffer, PBS and water, and mounted with fluorescence mounting medium (Dako, Agilent S3023). The observations were made on a Zeiss LSM710 confocal microscope using a CApochromat 40X NA 1.2 water immersion objective.

RT-qPCR. RNA was extracted using the TRIzol method (Invitrogen) and reverse transcribed with Superscript II reverse transcriptase (Invitrogen) according to the manufacturer's instructions. Quantitative real-time RT-PCR was performed using the SYBR Green Master mix kit (ThermoFisher). Primer sequences were as follows:

RPL32 (F) ACATCGGTTATGGGAGCAAC; RPL32 (R) TCCAGCTCCTTGACATTGT; IL$1 \beta$ (F) CAAGCTTCCTTGTGCAAGTG; IL-1ß (R) AGGTGGCATTTCACAGTTGA; IL-10 (F) CCTGGGTGAGAAGCTGAAGA; IL-10 (R) GCTCCACTGCCTTGCTCTTA; IFN-y (F) TGCCAAGTTTGAGGTCAACA; IFN-y (R) GAATCAGCAGCGACTCCTTT; IL-6 (F) 
GTTCTCTGGGAAATCGTGGA; IL-6 (R) GCAAGTGCATCATCGTTGTT; IL-17A (F) ATCCCTCAAAGCTCAGCGTGTC; IL-17A (R) GGGTCTTCATTGCGGTGGAGAG; IL$12 \mathrm{p} 35$ CCTCAGTTTGGCCAGGGTC;

IL-12p35 CAGGTTTCGGGACTGGCTAAG; IL-12p40 (F) ATGTGTCCTCAGAAGCTAACC; IL$12 \mathrm{p} 40$ CTAGGATCGGACCCTGCAGGGAAC;

TNF- $\alpha$ GCCTCCCTCTCATCAGTTCTA; TNF-a (R) GCTACGACGTGGGCTACAG; PHD2 (F) AGGCTATGTCCGTCACGTTG; PHD2 (R) TACCTCCACTTACCTTGGCG; HIF1a (F) CATCAGTTGCCACTTCCCCA; HIF1 $\alpha$ (R) GGCATCCAGAAGTTTTCTCACAC; HIF2 $\alpha$ (F) ACGGAGGTCTTCTATGAGTTGGC; HIF2a (R) GTTATCCATTTGCTGGTCGGC.

RNA Sequencing and analysis. All RNA-Seq analyses were performed using $\geq 2$ biological replicates. Total RNA was prepared from purified splenic Treg cells using the TRIzol method (Invitrogen). 200 ng of total RNA was subsequently used to prepare RNA-Seq library by using TruSeq RNA sample prep kit (Illumina) according to manufacturer's instructions. Paired-end RNA sequencing was performed on a Novaseq 6000 (Illumina) (BRIGHTcore joint facility, ULB-VUB, Brussels, Belgium). Sequenced reads were aligned to the mouse genome (NCBI37/mm9) and uniquely mapped reads were used to calculate gene expression. Data analysis was performed using $\mathrm{R}$ program (Deseq2 package). Differentially expressed genes are considered significant when the FDR (false discovery rate or adjusted p-value) $<0.05$ and the $\log _{2} \mathrm{FC}$ (fold change) $>$ 0.5 . Upstream regulators analysis was performed following Ingenuity pathway analysis (IPA). IPA predicts functional regulatory networks from gene expression data and provides a significance score ( $p$-value) for each network according to the fit of the network to the set of genes in the database.

Statistical analysis. All statistical analyses were conducted using GraphPad Prism (GraphPad Software). Statistical difference between two groups was determined by an unpaired, two-tailed student's $t$ tests. A one-way or two-way ANOVA was used for multigroup comparisons together with Tukey's multiple comparisons post hoc tests. Survival significance in DSS-induced colitis was determined by a Log-rank Mantel-Cox test. Data is judged to be statistically significant when $p$ value $<0.05$. In figures, asterisks denote statistical significance $\left({ }^{*}, p<0.05 ;{ }^{* *}, p<0.01\right.$; ${ }^{* * *}, p<0.001 ;{ }^{* * *}, p<$ 0.0001). 


\section{References}

1. Lu, L., Barbi, J. \& Pan, F. The regulation of immune tolerance by FOXP3. Nat. Rev. Immunol. 17, 703-717 (2017).

2. Xing, Y. \& Hogquist, K. A. T-Cell Tolerance: Central and Peripheral. Cold Spring Harb Perspect Biol 4, 1-15 (2012).

3. Dominguez-Villar, M. \& Hafler, D. A. Regulatory T cells in autoimmune disease. Nat. Immunol. 19, 665-673 (2018).

4. Samstein, R. M., Josefowicz, S. Z., Arvey, A., Treuting, P. M. \& Rudensky, A. Y. Extrathymic generation of regulatory $T$ cells in placental mammals mitigates maternal-fetal conflict. Cell 150, 29-38 (2012).

5. Togashi, Y., Shitara, K. \& Nishikawa, H. Regulatory T cells in cancer immunosuppression - implications for anticancer therapy. Nat. Rev. Clin. Oncol. (2019). doi:10.1038/s41571-019-0175-7

6. Aandahl, E. M., Michaëlsson, J., Moretto, W. J., Hecht, F. M. \& Nixon, D. F. Human CD4+ CD25+ Regulatory T Cells Control T-Cell Responses to Human Immunodeficiency Virus and Cytomegalovirus Antigens. J. Virol. 78, 2454-2459 (2004).

7. Van Der Burg, S. H. et al. Association of cervical cancer with the presence of CD4+ regulatory $T$ cells specific for human papillomavirus antigens. PNAS 104, 12087-12092 (2007).

8. Caridade, M., Graca, L. \& Ribeiro, R. M. Mechanisms underlying CD4+ Treg immune regulation in the adult: From experiments to models. Front. Immunol. 4, 1-9 (2013).

9. Clement, R. L. et al. Follicular regulatory T cells control humoral and allergic immunity by restraining early B cell responses. Nat. Immunol. 20, 1360-1371 (2019).

10. Vignali, D. A. A., Collison, L. W. \& Workman, C. J. How regulatory T cells work. Nat. Rev. Immunol. 8, 523-532 (2008).

11. Wardell, C. M., MacDonald, K. N., Levings, M. K. \& Cook, L. Cross talk between human regulatory $\mathrm{T}$ cells and antigen-presenting cells: Lessons for clinical applications. Eur. J. Immunol. 51, 27-38 (2021).

12. Shevyrev, D. \& Tereshchenko, V. Treg Heterogeneity, Function, and Homeostasis. Front. Immunol. 10, 1-13 (2020).

13. Panduro, M., Benoist, C. \& Mathis, D. Tissue Tregs. Annu. Rev. Immunol. 34, 609-633 (2016).

14. Ast, T. \& Mootha, V. K. Oxygen and mammalian cell culture: are we repeating the experiment of Dr. Ox? Nat. Metab. 1, 858-860 (2019).

15. McKeown, S. R. Defining normoxia, physoxia and hypoxia in tumours Implications for treatment response. Br. J. Radiol. 87, 1-12 (2014).

16. Bartels, K., Grenz, A. \& Eltzschig, H. K. Hypoxia and inflammation are two sides of the same coin. PNAS 110, 18351-18352 (2013).

17. Nanduri, J. et al. HIF-1 $\alpha$ activation by intermittent hypoxia requires NADPH oxidase stimulation by xanthine oxidase. PLoS One 10, 1-12 (2015).

18. Mempel, T. R. \& Marangoni, F. Guidance factors orchestrating regulatory T cell positioning in tissues during development, homeostasis, and response. Immunol. 
Rev. 289, 129-141 (2019).

19. Hirota, K. Basic biology of hypoxic responses mediated by the transcription factor HIFs and its implication for medicine. Biomedicines 8, (2020).

20. Baik, A. H. \& Jain, I. H. Turning the Oxygen Dial: Balancing the Highs and Lows. Trends Cell Biol. 30, 516-536 (2020).

21. Wang, G. L. \& Semenza, G. L. Purification and characterization of hypoxiainducible factor. Journal of Biological Chemistry 270, 1230-1237 (1995).

22. J.D., W., M.L., C. \& C.W., P. Hypoxia, hypoxia-inducible factors (HIF), HIF hydroxylases and oxygen sensing. Cell. Mol. Life Sci. 66, 3539-3554 (2009).

23. Semenza, G. L. HIF-1, O2, and the 3 PHDs: How animal cells signal hypoxia to the nucleus. Cell 107, 1-3 (2001).

24. Maxwell, P. H. et al. The tumoursuppressor proteinVHLtargets hypoxia-inducible factors for oxygen-dependent proteolysis. Nature 44, 271-275 (1999).

25. Downes, N. L., Laham-Karam, N., Kaikkonen, M. U. \& Ylä-Herttuala, S. Differential but Complementary HIF $1 \alpha$ and HIF2 $\alpha$ Transcriptional Regulation. Mol. Ther. 26, 1735-1745 (2018).

26. Watts, E. R. \& Walmsley, S. R. Inflammation and Hypoxia: HIF and PHD Isoform Selectivity. Trends Mol. Med. 25, 33-46 (2019).

27. Talks, K. L. et al. The expression and distribution of the hypoxia-inducible factors HIF-1 $\alpha$ and HIF-2 $\alpha$ in normal human tissues, cancers, and tumor-associated macrophages. Am. J. Pathol. 157, 411-421 (2000).

28. Hu, C.-J., Sataur, A., Wang, L., Chen, H. \& Simon, M. C. The N-Terminal Transactivation Domain Confers Target Gene Specificity of Hypoxia-inducible Factors HIF-1a and HIF-2a. Mol. Biol. Cell 18, 986-994 (2007).

29. Meneses, A. M. and \& Wielockx, B. PHD2 : from hypoxia regulation to disease progression. Dovepress 53-67 (2016).

30. Corrado, C. \& Fontana, S. Hypoxia and HIF signaling: One axis with divergent effects. Int. J. Mol. Sci. 21, 1-17 (2020).

31. Wohlfert, E. A., Warunek, J., Jin, R. M. \& Marzullo, B. Tbet - expressing Tregs protect against lethal immunopathology during $\mathrm{T}$. gondii infection. J Immunol 204, 2020 (2020).

32. Esplugues, E. et al. Control of TH17 cells occurs in the Small Intestine. Nature 475, 514-518 (2012).

33. Koch, M. A. et al. The transcription factor T-bet controls regulatory T cell homeostasis and function during type 1 inflammation. Nat. Immunol. 10, 595-602 (2009).

34. Hall, A. O. H. et al. The Cytokines Interleukin 27 and Interferon-y Promote Distinct Treg Cell Populations Required to Limit Infection-Induced Pathology. Immunity 37, 511-523 (2012).

35. Barbi, J., Oghumu, S., Lezama-Davila, C. M. \& Satoskar, A. R. IFN-ץ and STAT1 are required for efficient induction of $\mathrm{CXC}$ chemokine receptor 3 (CXCR3) on CD4+ but not CD8+ T cells. Blood 110, 2215-2216 (2007).

36. Fontenot, J. D., Gavin, M. A. \& Rudensky, A. Y. Foxp3 programs the development and function of CD4+CD25+ regulatory T cells. J. Immunol. 4, 986-992 (2003).

37. Redpath, S. A. et al. ICOS controls Foxp3+ regulatory T-cell expansion, maintenance and IL-10 production during helminth infection. Eur. J. Immunol. 43, 705-715 (2013). 
38. Lowther, D. E. et al. PD-1 marks dysfunctional regulatory T cells in malignant gliomas. JCI 1, 1-15 (2016).

39. Tan, C. L. et al. PD-1 restraint of regulatory T cell suppressive activity is critical for immune tolerance. J. Exp. Med. 218, (2021).

40. Franckaert, D. et al. Promiscuous Foxp3-cre activity reveals a differential requirement for CD28 in Foxp3 + and Foxp3-T cells. Immunol. Cell Biol. 93, 417423 (2015).

41. Yamamoto, A. et al. Systemic silencing of Phd2 causes reversible immune regulatory dysfunction. J. Clin. Invest. 129, 3640-3656 (2019).

42. Levine, A. G. et al. Stability and function of regulatory T cells expressing the transcription factor T-bet. Nature 546, 421-425 (2017).

43. Littringer, K. et al. Common features of regulatory T cell specialization during Th1 responses. Front. Immunol. 9, 1-15 (2018).

44. Duckworth, B. C. et al. Effector and stem-like memory cell fates are imprinted in distinct lymph node niches directed by CXCR3 ligands. Nat. Immunol. (2021). doi:10.1038/s41590-021-00878-5

45. Biasci, D. et al. CXCR4 inhibition in human pancreatic and colorectal cancers induces an integrated immune response. PNAS 117, 28960-28970 (2020).

46. Clever, D. et al. Oxygen Sensing by T Cells Establishes an Immunologically Tolerant Metastatic Niche. Cell 166, 1117-1131.e14 (2016).

47. Hsu, T. S. et al. HIF-2 $\alpha$ is indispensable for regulatory T cell function. Nat. Commun. 11, 1-16 (2020).

48. Clambey, E. T. et al. Hypoxia-inducible factor-1 alpha - dependent induction of FoxP3 drives regulatory T-cell abundance and function during inflammatory hypoxia of the mucosa. PNAS 109, (2012).

49. Lee, J. H., Elly, C., Park, Y. \& Liu, Y. C. E3-Ubiquitin Ligase VHL Regulates Hypoxia-Inducible Factor-1a to Maintain Regulatory T Cell Stability and Suppressive Capacity. Immunity 42, 1062-1074 (2015).

50. Shi, H. \& Chi, H. Metabolic Control of Treg Cell Stability, Plasticity, and TissueSpecific Heterogeneity. Front. Immunol. 10, 1-17 (2019).

51. Gupta, N. \& Wish, J. B. Hypoxia-Inducible Factor Prolyl Hydroxylase Inhibitors: Potential New Treatment for Anemia in Patients With CKD. Am. J. Kidney Dis. 69, 815-826 (2017).

52. Marks, E. et al. Oral Delivery of Prolyl Hydroxylase Inhibitor: AKB-4924 Promotes Localized Mucosal Healing in a Mouse Model of Colitis. Inflamm Bowel Dis 21, 267-275 (2015).

53. Li, X. et al. Therapeutic Potential of a Prolyl Hydroxylase Inhibitor FG-4592 for Parkinson's Diseases in Vitro and in Vivo : Regulation of Redox Biology and Mitochondrial Function. Frontiers (Boulder). 10, 1-16 (2018).

54. Rubtsov, Y. P. et al. Regulatory T Cell-Derived Interleukin-10 Limits Inflammation at Environmental Interfaces. Immunity 28, 546-558 (2008).

55. Kim, J. J., Shajib, S., Manocha, M. M. \& Khan, W. I. Investigating Intestinal Inflammation in DSS-induced Model of IBD. Jove 1, 1-6 (2012). 


\section{Acknowledgments}

We thank Valérie Acolty, Caroline Abdelaziz and Véronique Dissy for animal care and for technical support. We also wish to thank Sébastien Denanglaire for his help in cell purification by flow cytometry. The development of mouse models of toxoplasmosis would not have been possible without the kind assistance of Guillaume Oldenhove. This work was supported by the European Regional Development Fund (ERDF) and the Walloon Region (Wallonia-Biomed portfolio, 411132- 957270), a grant from the Fonds Jean Brachet and research credit from the National Fund for Scientific Research, FNRS, Belgium. The CMMI is supported by the European Regional Development Fund and the Walloon Region. FA is a Research Associate at the FNRS. YA is recipient of a research fellowship from the FNRS/Télévie. $\mathrm{HH}$ has been supported by a Belgian FRIA fellowship.

\section{Author contributions statement}

YA performed most of the experiments, acquired and analyzed the data. AT and HH performed additional experiments and contributed to data analysis. AA performed the bioinformatic analysis. SG, FA and MM provided useful reagents and supervised the research program. OL conceived and supervised the research program and wrote the manuscript with input from all co-authors.

\section{Additional information}

The authors declare that the research was conducted in the absence of any commercial or financial relationships that could be construed as a potential conflict of interest. 


\section{Figure legends}

Fig. 1 PHD2 ${ }^{\Delta \text { Treg }}$ mice display a spontaneous Th1-like inflammatory syndrome. a Body weight of naive mice was determined weekly. b At 12 weeks of age, mice were examined for rectal prolapse c Representative gross autopsy of an hemorrhagic abdomen, $\mathbf{d}$ splenomegaly and colon length summarized in $\mathbf{e .}(\mathbf{f}-\mathbf{i})$ Lymphoid cells from spleen, mesenteric $(\mathrm{mLN})$, peripheral $(\mathrm{pLN})$ lymph nodes or the small intestine lamina propria were collected from Foxp ${ }^{\text {cre }}$ and $\mathrm{PHD} 2^{\Delta \text { Treg }}$ mice. $f$ Frequency of conventional, Foxp3- CD4 and CD8-expressing cells among TCR 3 -expressing T lymphocytes. g Representative merged $(n=15)$ t-distributed stochastic neighbor embedding (t-SNE) plot after dimensionality reduction and unsupervised clustering of flow cytometry data from CD4-expressing spleen cells. Relative distributions of $\mathrm{CD}^{+}$lymphocyte subsets are shown as doughnut charts. $h$ Frequency of effector-like (CD44 ${ }^{\text {hi }}$ CD62 $^{\text {lo }}$ ) conventional T lymphocytes in the indicated lymphoid organs. i Frequency of IFN-y (top panel) and IL-17A (bottom panel) producing $\mathrm{CD}^{+} \mathrm{T}$ cells after in vitro stimulation. $\mathbf{j}$ Expression of inflammatory cytokines determined by qPCR on extracts from unfractionated $\mathrm{mLNs}$. Data are representative of at least three independent experiments with $n=9(\mathbf{a}, \mathbf{j}), n=25(\mathbf{e}), n=15(\mathbf{f}-\mathbf{i})$ per group. Values are presented as the mean \pm standard deviation (SD) and were compared by two-tailed unpaired student's t-test. Only significant differences are indicated as follows: ${ }^{*}: p<0.05,{ }^{* *}: p<0.01,{ }^{* * *}: p<0.001,{ }^{* * * *}$ : $\mathrm{p}<0.0001$. Abbreviations: Naive Tconv (Foxp3 ${ }^{-} \mathrm{CD}^{-} 4^{-} \mathrm{CD} \mathrm{CL}^{+}{ }^{+}$), Memory Tconv (Foxp3 ${ }^{-}$ $\mathrm{CD}_{4}{ }^{+} \mathrm{CD} \mathrm{CL}^{+}$), Effector Tconv (Foxp3 ${ }^{-} \mathrm{CD}_{4}{ }^{+} \mathrm{CD}^{-} \mathrm{L}^{-}$), Memory Treg (Foxp3 ${ }^{+}$ $\mathrm{CD}_{4} 4^{+} \mathrm{CD} 62 \mathrm{~L}^{+}$), Effector Treg (Foxp3 ${ }^{+} \mathrm{CD}_{4} 4^{+} \mathrm{CD} 6 \mathrm{~L}^{-}$).

Fig. 2 Increased number, but altered phenotype of PHD2-deficient Treg cells Lymphoid cells from spleen, mesenteric $(\mathrm{mLN})$ and peripheral $(\mathrm{pLN})$ lymph nodes were collected at 12 weeks of age from Foxp3 $3^{\text {cre }}$ and $\mathrm{PHD}^{\Delta \mathrm{Treg}}$ mice and the relative frequency and phenotype of Foxp3-expressing cells were established by flow cytometry or qPCR. a Frequency of Foxp3-expressing cells among CD4-positive T lymphocytes; $\mathbf{b}$ frequency of effector (CD62 ${ }^{\text {low }} \mathrm{CD} 44^{\text {high }}$ ), memory (CD62L ${ }^{\text {high }} \mathrm{CD} 44^{\text {high }}$ ) and naive (CD62L $\mathrm{L}^{\text {high }} \mathrm{CD} 44^{\text {low }}$ ) Foxp3-expressing cells; c frequency of Tregs expressing the master transcription factors T-bet, GATA3 and RORyt; d Ratio of the Foxp3 MFI of PHD2-KO splenic Tregs to Foxp3 ${ }^{\text {cre }}$ splenic Tregs. e Expression of CD25, CD44, ICOS, PD-1 and CTLA-4 in splenic Treg of Foxp3 ${ }^{\text {cre }}$ and PHD2 ${ }^{\Delta T r e g}$ mice. Top panel, representative traces of MFI. Bottom panel, ratios of the MFIs of PHD2-KO Treg to Foxp3 ${ }^{\text {cre }}$ Treg cells are expressed as the mean \pm SD. $f \mathrm{IL}-10$ gene expression relative to RPL32 by ex-vivo purified Tregs was determined by qPCR. (g-i) $\mathrm{CD}^{+}{ }^{+}$Foxp3 ${ }^{-}$splenic naive $T$ cells were stimulated in vitro with anti-CD3/CD28 $\left(5 / 1 \mu \mathrm{g} \mathrm{ml}^{-1}\right)$ in the presence of TGF- $\beta$ (3 $\left.\mu \mathrm{g} \mathrm{ml}^{-1}\right)$ and IL-2 $\left(10 \mu \mathrm{g} \mathrm{ml}^{-1}\right)$ for $72 \mathrm{~h}$ to induce Treg polarization. $\mathrm{g}$ Representative flow cytometry expression profiles of Foxp3 expression at the end of the culture period. The first panel represents a typical profile of cells activated in the absence of polarizing cytokines; $\boldsymbol{h}$ number of Foxp $3^{+}$cells generated in the culture conditions; i Expression (MFI) of Foxp3 by in vitro induced Treg cells. Data are representative of at least three independent experiments with $n=15(\mathbf{a}-\mathbf{d}), n=9$ (e-f) or $n=6(h-i)$ per group. Values are presented as the mean \pm SD and were compared by 
two-tailed unpaired student's t-test. Only significant differences are indicated as follows: *: $p<0.05,{ }^{* *}: p<0.01,{ }^{* * *}: p<0.001$.

\section{Fig. 3 Reduced in vivo but not in vitro suppressive capacity of PHD2-deficient Treg.}

a Treg function was assayed following adoptive co-transfer of CD45.2 Foxp3-expressing cells with naive, CFSE labeled congenic CD45.1 CD4 ${ }^{+}$lymphocytes (Treg : Tconv ratio $1: 3)$ into syngeneic lymphopenic mice $\left(\operatorname{Rag}_{2}{ }^{--}\right)$. Recipient mice were euthanized at day 6 post-transfer, and their spleen and $\mathrm{mLN}$ cells analyzed by flow cytometry. b Representative flow cytometry expression profiles of CFSE labeled cells (CD45.1 gate in the spleen) with or without co-transferred Foxp $3^{+}$cells from Foxp $3^{\text {cre }}$ or PHD2 ${ }^{\Delta T r e g}$ mice; c percentage of suppression established from CFSE staining profiles; d frequency of activated $\left(\mathrm{CD} 4^{+} \mathrm{CD} 45.1^{+} \mathrm{CD}_{4} 4^{\mathrm{hi}} \mathrm{CD}_{2} \mathrm{~L}^{\mathrm{lo}}\right.$ ) cells in the indicated lymphoid organs; $\mathbf{e}$ frequency of Treg cells in the indicated organs 6 days post-transfer. (f, g) CFSE-labeled, naive conventional $\mathrm{CD}^{+} \mathrm{T}$ cells from CD54.1 mice were co-cultured with ex-vivo purified Treg cells from Foxp $3^{\text {cre }}$ or PHD2 ${ }^{\Delta \text { Treg }}$ mice at the indicated ratios in the presence of antiCD3 antibodies $\left(0.5 \mu \mathrm{g} \mathrm{ml}^{-1}\right)$ and splenic feeder cells; $\mathbf{f}$ representative flow cytometry profiles of CSFE staining; $\mathbf{g}$ Percent of suppression of proliferation as compared to cultures in which Treg cells were omitted. Data are representative of three independent experiments with $n=15$ (b-e) or $n=4(\mathbf{f}, \mathbf{g})$ per group. Values are presented as the mean \pm SD and were compared by two-way ANOVA with Tukey's multiple comparisons test (c-e) or by two-tailed unpaired student's t-test $(\mathbf{g})$. Only significant differences are indicated as follows: ${ }^{*}: p<0.05,{ }^{* *}: p<0.01,{ }^{* * *}: p<0.001$.

Fig. 4 Increased sensitivity of $\mathrm{PHD}^{\Delta \mathrm{Treg}}$ mice to DSS-induced colitis and toxoplasmosis.

Foxp3 ${ }^{\text {cre }}$ and PHD2 ${ }^{\Delta \text { Treg }}$ mice were provided with $2 \%$ DSS in tap water for five days. On day 5 , the $2 \%$ DSS water was replaced with normal drinking water and mice were followed during 14 days for a body weight, b survival, c colitis severity and d colon length. e Colons were isolated from untreated mice or 6 days after colitis induction and were fixed and stained with hematoxylin and eosin (H\&E); arrows indicate inflammatory cell infiltrates. f Foxp $3^{\text {cre }}$ and $\mathrm{PHD} 2^{\Delta \text { Treg }}$ mice were infected by intragastric gavage with 10 cysts of ME-49 type II Toxoplasma gondii (control group are Foxp3 ${ }^{\text {cre }}$ mice without treatment) and subsequently followed for $\mathbf{g}$ body weight. $\mathbf{h}$ Mice were sacrificed 8 days after infection to assess colon length; i frequency of effector-like (CD44 ${ }^{\text {hi }} \mathrm{CD} 2 \mathrm{~L}^{\text {lo }}$ ) conventional $\mathrm{T}$ lymphocytes in the indicated lymphoid organs; $\mathbf{j}$ frequency of IFN- $\mathrm{Y}$ producing $\mathrm{CD}^{+}{ }^{+} \mathrm{T}$ cells after in vitro stimulation; $\mathbf{k}$ frequency of $\mathrm{T}$-bet ${ }^{+}$among Foxp $3^{+}$(Treg) cells. Data are representative of three independent experiments with $n=$ $20(\mathbf{a}, \mathbf{b}), \mathrm{n}=10-14(\mathbf{c}, \mathbf{d}), \mathrm{n}=5(\mathbf{e})$ or $\mathrm{n}=10(\mathbf{g}-\mathbf{k})$ per group. Values are presented as the mean $\pm S D$ and were compared by two-tailed unpaired student's t-test $(\mathbf{a}, \mathbf{c}, \mathbf{g})$, by Mantel-Cox test (b), by one-way ANOVA with Tukey's multiple comparisons test (d, $\mathbf{h}$ ) or by two-way ANOVA with Tukey's multiple comparisons test (i-k). Only significant differences are indicated as follows: ${ }^{*}: p<0.05,{ }^{* *}: p<0.01,{ }^{* * *}: p<0.001,{ }^{* * * *}: p<0.0001$. 
Fig. 5 Concomitant loss of HIF2 $\alpha$ but not HIF1a expression attenuates the proinflammatory phenotype of PHD2 ${ }^{\Delta \text { Treg }}$ mice.

a Representative gross autopsy of spleens and colon length summarized in $\mathbf{b}$ of Foxp3 $^{\text {cre }}$, PHD2 $^{\Delta \text { Treg }}$, PHD2-HIF1 $\alpha^{\Delta \text { Treg }}$, PHD2-HIF2 $\alpha^{\Delta \text { Treg }}$ and PHD2-HIF1 $\alpha-H I F 2 \alpha^{\Delta T r e g}$ (TKO) mice. c Representative merged $(n=15)$-distributed stochastic neighbor embedding (t-SNE) plot after dimensionality reduction and unsupervised clustering of flow cytometry data from CD4-expressing spleen cells. Relative distributions of CD4 ${ }^{+}$ lymphocyte subsets are shown as doughnut charts. (d-g) Lymphoid cells from spleen, mesenteric $(\mathrm{mLN})$, peripheral $(\mathrm{pLN})$ lymph nodes or the small intestine lamina propria were collected from Foxp3 ${ }^{\text {cre }}, \mathrm{PHD}^{\Delta \text { Treg }}$, PHD2-HIF $1 \alpha^{\Delta \text { Treg }}$ mice, PHD2-HIF2 $\alpha^{\Delta \text { Treg }}$ and PHD2-HIF1 $\alpha-H I F 2 \alpha^{\Delta T r e g}($ TKO) mice and the relative frequency and phenotype of Foxp3positive and Foxp3-negative, conventional T lymphocytes determined by flow cytometry. d Frequency of effector-like $\left(\mathrm{CD} 44^{\mathrm{hi}} \mathrm{CD} 2 \mathrm{~L}^{\mathrm{lo}}\right)$ conventional $\mathrm{T}$ lymphocytes in the indicated lymphoid organs. e Frequency of IFN- $\gamma$ producing $\mathrm{CD}^{+}{ }^{+} \mathrm{T}$ cells after in vitro stimulation. f Frequency of Foxp3-expressing cells among CD4-positive T lymphocytes. g Ratio of the Foxp3 MFI of PHD2-KO, PHD2-HIF1aKO, PHD2-HIF2aKO or TKO splenic Tregs to Foxp3 ${ }^{\text {cre }}$ splenic Tregs. Data are representative of at least three independent experiments with $n=15$ per groups. Values are expressed as the mean \pm SD and were compared by One-way ANOVA with Tukey's multiple comparisons test (b, g) or by Two-way ANOVA with Tukey's multiple comparisons test (d-f). Only significant differences are indicated as follows: ${ }^{*}: p<0.05,{ }^{* *}: p<0.01,{ }^{* *}: p<0.001,{ }^{* * * *}: p<0.0001$.

Fig. 6 Anti-inflammatory response, response to chemokines and cell survival pathways represent targets of the PHD2-HIF2 $\alpha$ axis in Tregs.

Splenic Treg cells were purified by cell sorting from Foxp3 $3^{\text {cre }}(n=3), \operatorname{PHD}^{\Delta \operatorname{Treg}}(n=2)$, PHD2-HIF1 $\alpha^{\Delta \text { Treg }}(n=2)$, PHD2-HIF2 $^{\Delta{ }^{\Delta T r e g}}(n=3)$ and PHD2-HIF $1 \alpha-H I F 2 \alpha^{\Delta T r e g}($ TKO) $(n$ = 3) mice and total RNA was extracted and sequenced by RNA-sequencing (Illumina). a Heatmap of genes differentially expressed. Values are represented as $\log _{2}$ fold-change obtained from median of each gene and are plotted in red-blue color scale with red indicating increased expression and blue indicating decreased expression. Hierarchical clustering of genes ( $\mathrm{k}$ mean clustering) show 20 clusters. b Classification of mouse strains according to their spontaneous inflammation severity. c Heatmap of genes downregulated when PHD2 and PHD2-HIF1 $\alpha$ are deleted and whose expression is restored to a control level (close to Foxp3 ${ }^{\text {cre }}$ Treg) following deletion of HIF2a (Cluster 10, 181 genes). d Heatmap of genes upregulated when PHD2 and PHD2-HIF1a are deleted and whose expression is restored to a control level following deletion of HIF2 $\alpha$ (Cluster 11, 66 genes). e Heatmap of genes upregulated when PHD2 and PHD2-HIF2 $\alpha$ are deleted and whose expression is restored to a control level following deletion of HIF1a (Cluster 3, 98 genes). Cluster 3, 10 and 11 were subjected to functional annotations and regulatory network analysis in the Ingenuity Pathway Analysis (IPA) software. Data were analyzed using Deseq2, a gene is differentially expressed when $\log _{2} \mathrm{FC}$ (Fold change) $>0.5$ and FDR (False discovery rate) $<0.05$. 
Fig. 7 Identification of STAT1-mediated signaling as a target of the PHD2-HIF2 $\alpha$ axis in Tregs. a Upregulated and downregulated genes (cluster 10 and 11 in Fig. 6 c-d) were imported into the Ingenuity Pathway Analysis (IPA) software and were subjected to Upstream regulator analysis (URA) prediction algorithms. STAT1 was predicted as an upstream regulators of downregulated genes with a $p$-value $=3.10^{-12}$. Phosphorylated form of STAT1 (pSTAT1 (Tyr701)) was assessed by flow cytometry after brief in vitro stimulation (30 $\mathrm{min}$ ) of splenic $\mathrm{CD}^{+} \mathrm{T}$ lymphocytes with recombinant IFN- $\mathrm{y}$. b Representative histogram of pSTAT1 MFI for conventional CD4 ${ }^{+} \mathrm{T}$ cells (Tconv) and Treg cells of Foxp3 $3^{\text {cre }}$, PHD2 $^{\Delta \text { Treg }}$ and PHD2-HIF2 $a^{\Delta \text { Treg }}$ mice. Mean value expression (represented by MFI) of $\mathbf{c}$ pSTAT1 or d STAT1 total protein by splenic Treg of Foxp3 ${ }^{\text {cre }}$, $\mathrm{PHD}^{\triangle \mathrm{Treg}}$ and PHD2-HIF2 $\alpha^{\Delta T r e g}$ mice. Data are representative of three independent experiments with $n=9$ per groups. Values are presented as the mean \pm SD and were compared by one-way ANOVA with Tukey's multiple comparisons. Only significant differences are indicated as follows: ${ }^{* *}: p<0.01,{ }^{* * *}: p<0.001$.

Fig. 8 The PHD2-HIF2 $\alpha$ axis regulates adequate positioning of Tregs in the splenic white pulp.

a Frequency of Treg cells expressing the CXCR3 receptor. b Immunofluorescence of CD4 (red), and Foxp3 (green) in spleen sections of Foxp3 ${ }^{\text {cre }}, \mathrm{PHD}^{\Delta \mathrm{Treg}}$ and PHD2HIF2 $\alpha^{\Delta T r e g}$ mice. The functional regions of the spleen (1. red pulp, 2. white pulp and 3. marginal zone) were highlighted manually by an independent observer. The white line represents the marginal zone surrounding the white pulp. Treg cells have been manually highlighted by small white circles. c Frequency of Treg cells in the white and red pulp. $\mathbf{d}$ Average distance (in $\mu \mathrm{m}$ ) of Treg cells from the border of the white pulp (marginal zone). e Percentage of Treg located at a distance of less than $20 \mu \mathrm{m}$ from its nearest neighbor. The Nearest Neighbor Analysis measures the spread or distribution of cells over a geographical space. Data are representative of three (a) or two (b-e) independent experiments with $n=12(a)$ or $n=4$ (b-e) per groups. Values are presented as the mean \pm SD and were compared by two-way ANOVA with Tukey's multiple comparisons test (a, c) or by one-way ANOVA with Tukey's multiple comparisons test (d, e). Only significant differences are indicated as follows: ${ }^{*}: p<0.05,{ }^{* *}: p<0.01,{ }^{* * *}: p<0.001$. 
a (which was not certified by peer review) is the author/funder. All rights reserved. No reuse allowed without permission.

a

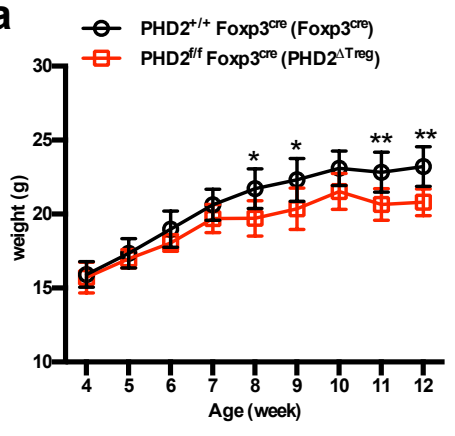

b

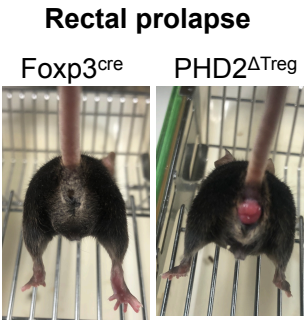

Hemorrhagic abdomen

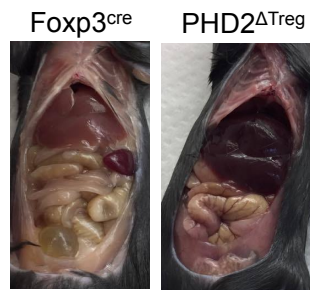

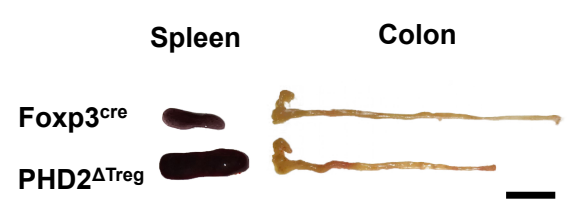

e
$\begin{array}{ll}\text { O } & \text { Foxp3 } \\ \text { cre } \\ \text { PHD2 }^{\Delta \text { Treg }}\end{array}$

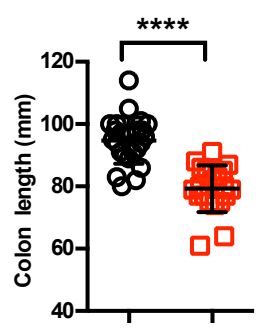

h

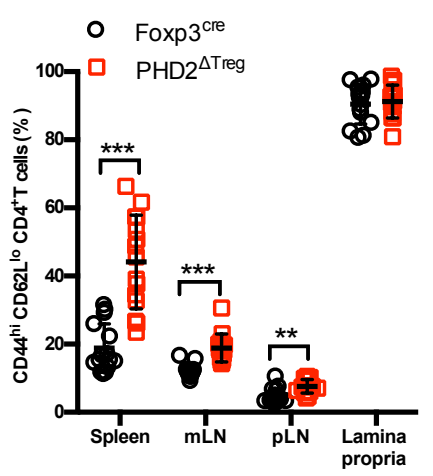

- Foxp $3^{\text {cre }}$
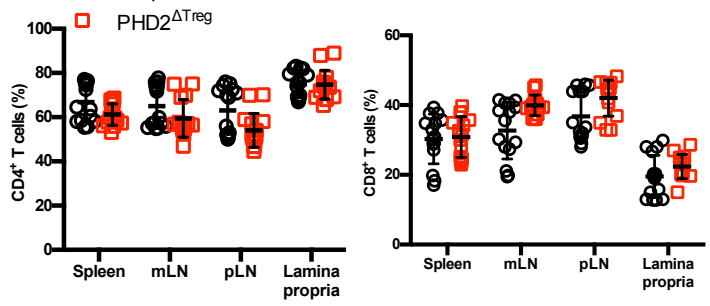

g
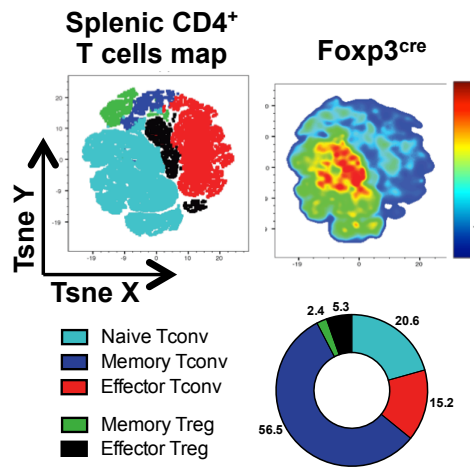

PHD2 $^{\Delta \text { Treg }}$

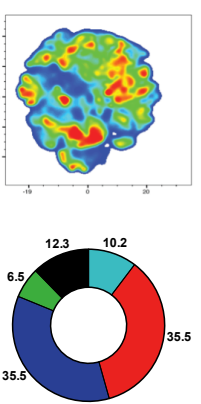

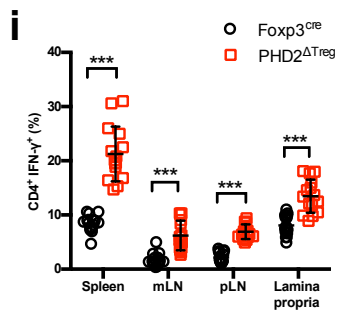

j $\circ$ Foxp3 $3^{\text {re }}$

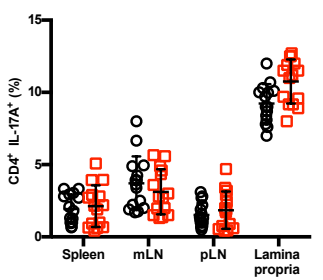

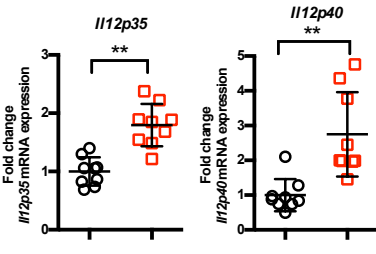
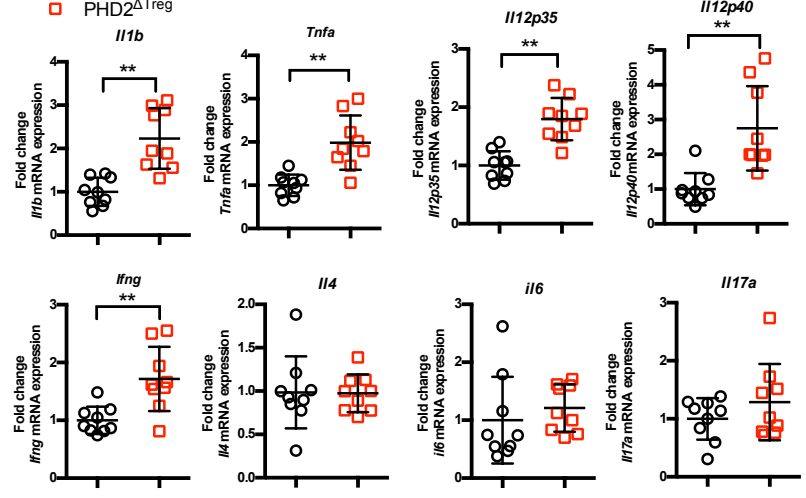

Fig. 1 PHD2 ${ }^{\Delta \text { Treg }}$ mice display a spontaneous Th1-like inflammatory syndrome. 


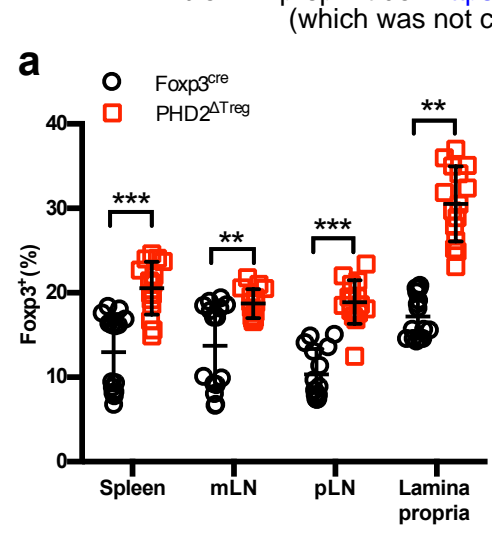

b c d
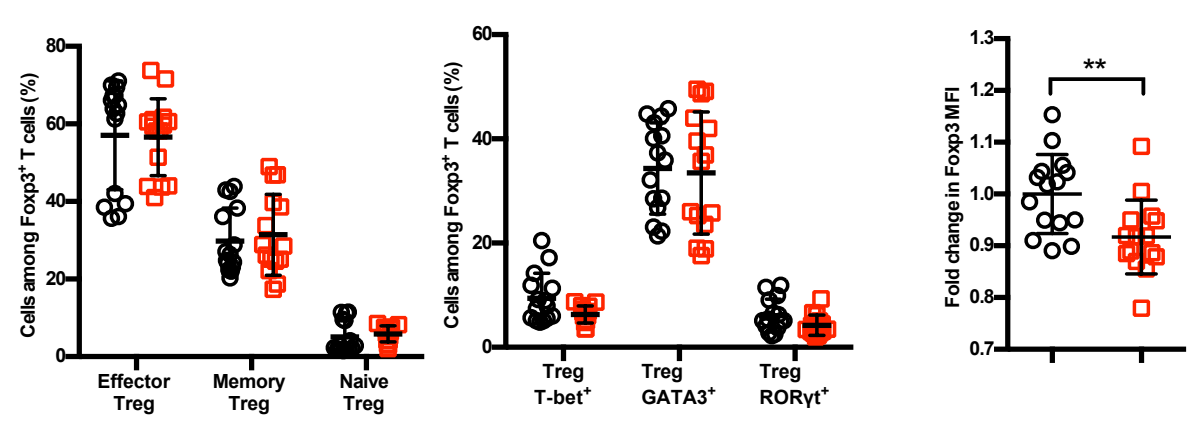

e

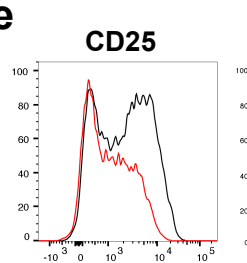

CD44
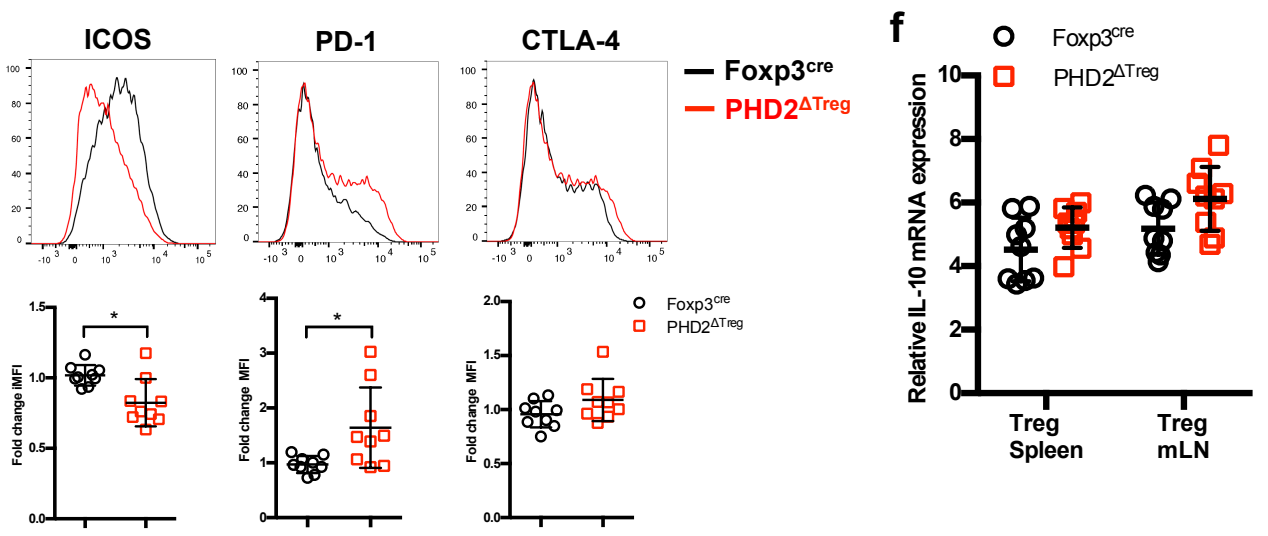

g
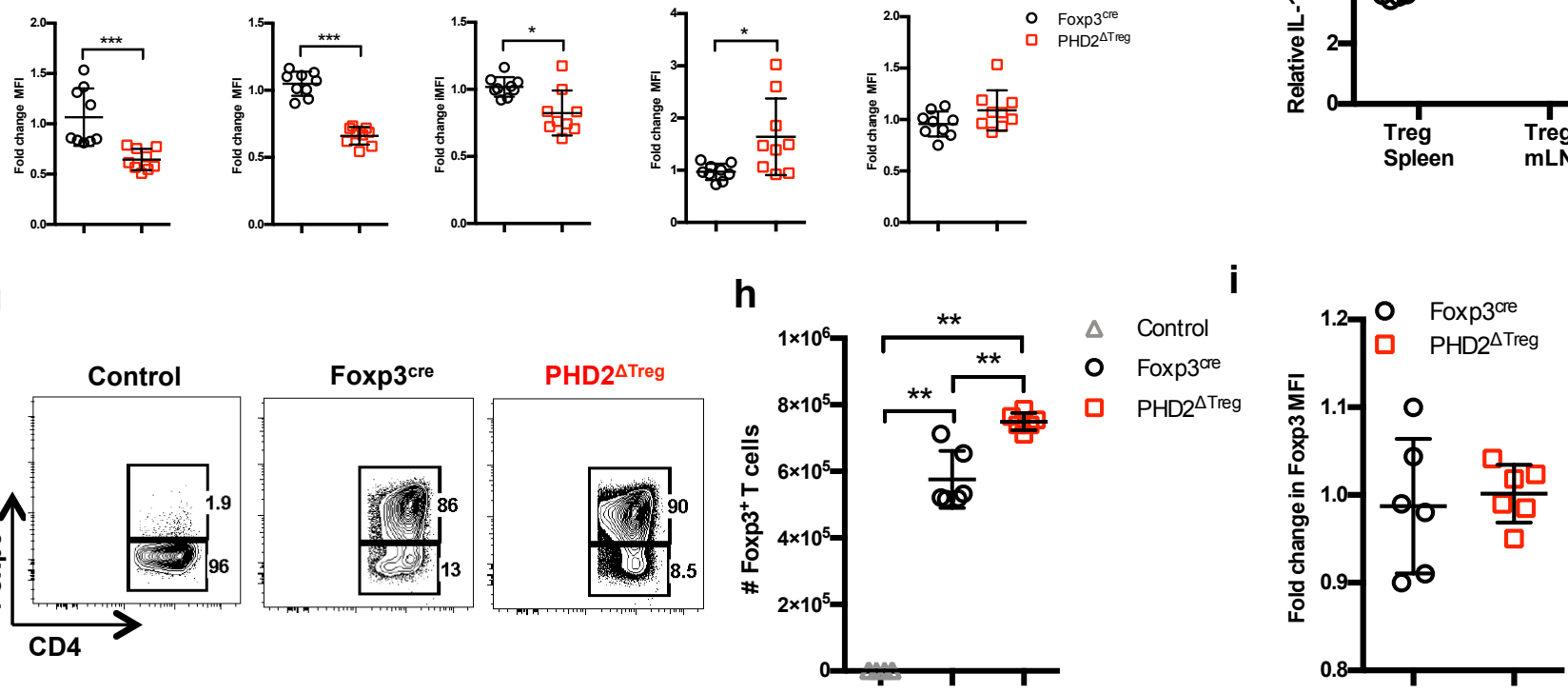

Fig. 2 Increased number, but altered phenotype of PHD2-deficient Treg cells 
a

$1 \times 10^{6} \mathrm{CD}^{+} \mathrm{CD} 25-$

CD45. $1^{+}$naive cells

CFSE-labeled

$+/-$

$3.3 \times 10^{5}$ of Foxp3 $3^{\text {cre }}$ or

PHD2-deficient Treg

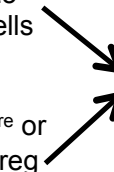

B6 Rag2-

Organ analysis

Day $0 \quad$ by flow cytom 6

C

$\Delta \quad$ No Treg

- Foxp3 ${ }^{\text {ree }}$ Treg

口 PHD2-deficient Treg

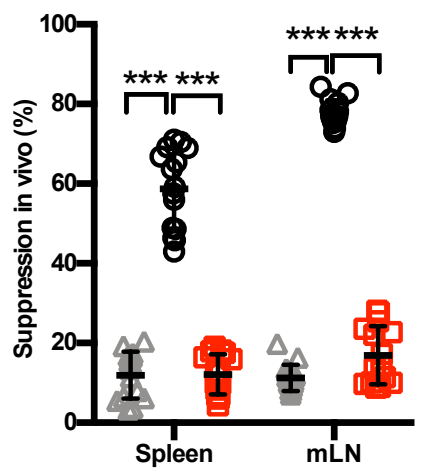

f

Treg:Tconv

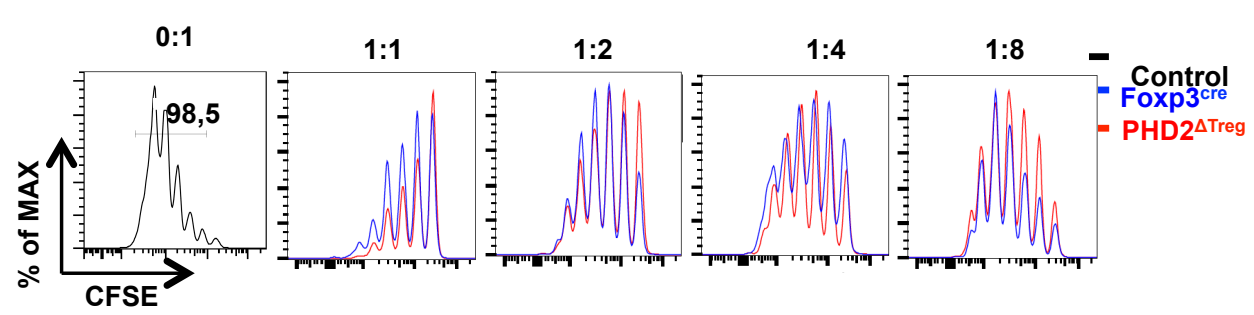

b

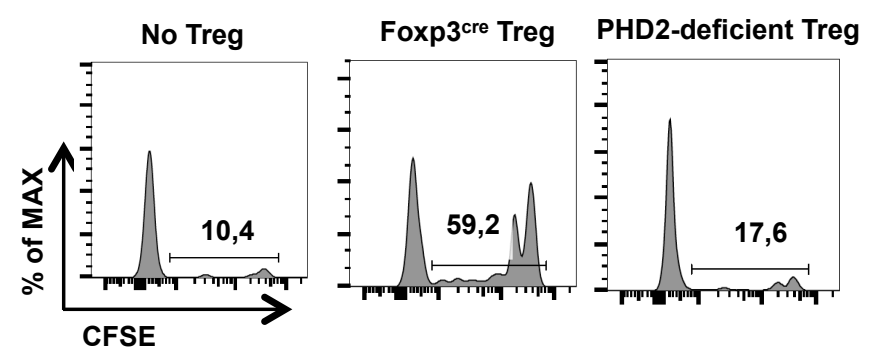

e
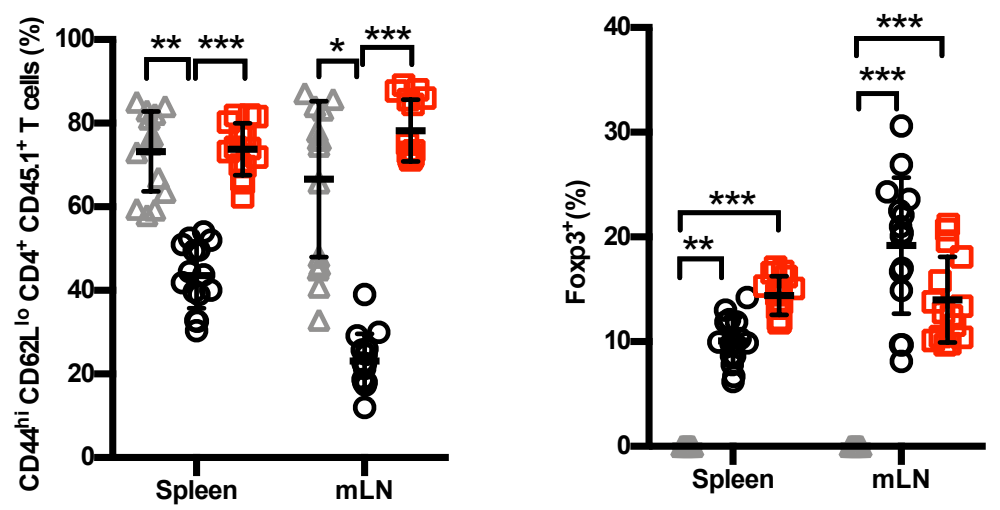

g

$\triangle$ No Treg O Foxp3 ${ }^{\text {cre }}$ Treg $\square$ PHD2-deficient Treg

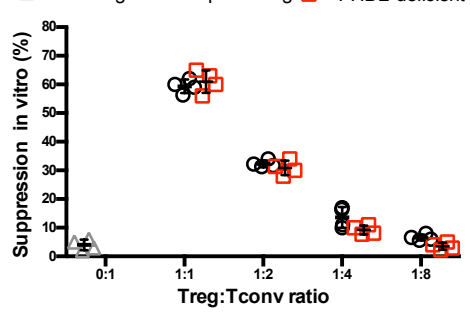

Fig. 3 Reduced in vivo but not in vitro suppressive capacity of PHD2-deficient Treg. 
(which was not certified by peer review) is the author/funder. All rights reserved. No reuse allowed without permission.

a $\quad$ Foxp3

$\because$ PHD2 $^{\Delta \text { Treg }}$

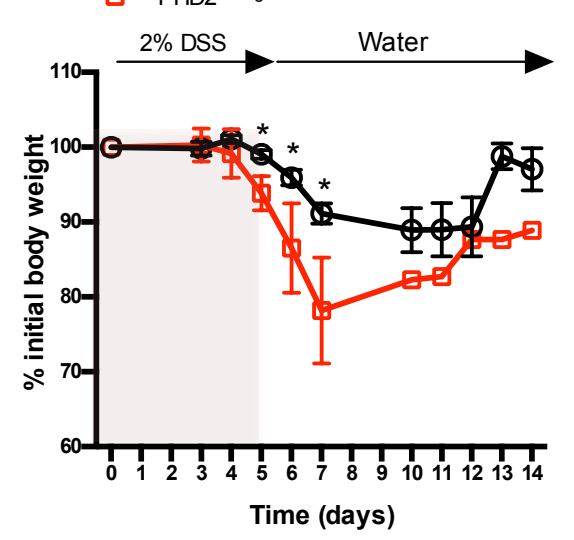

d

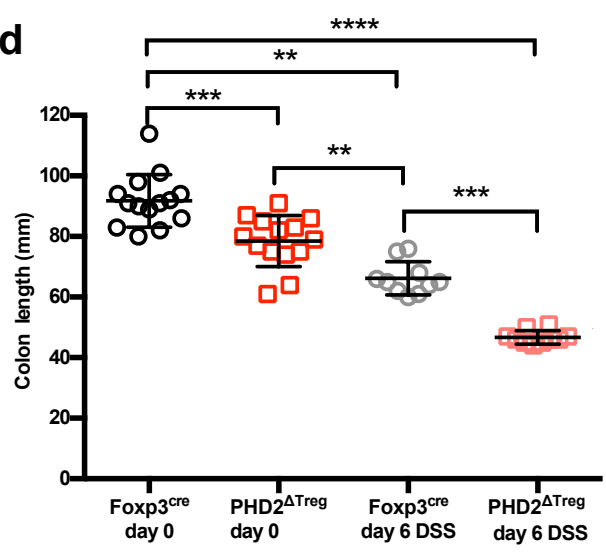

f

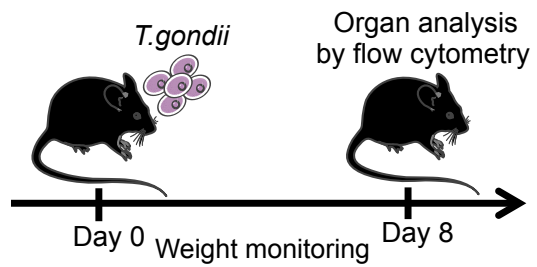

i

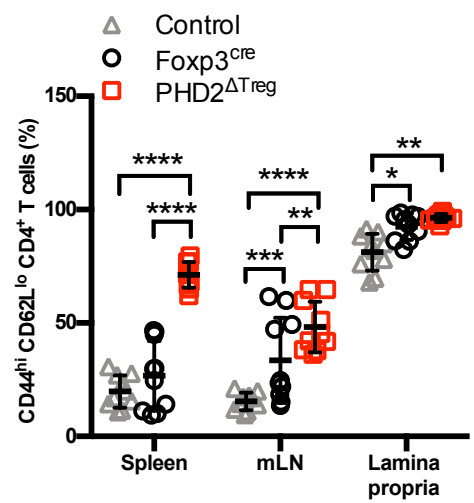

b

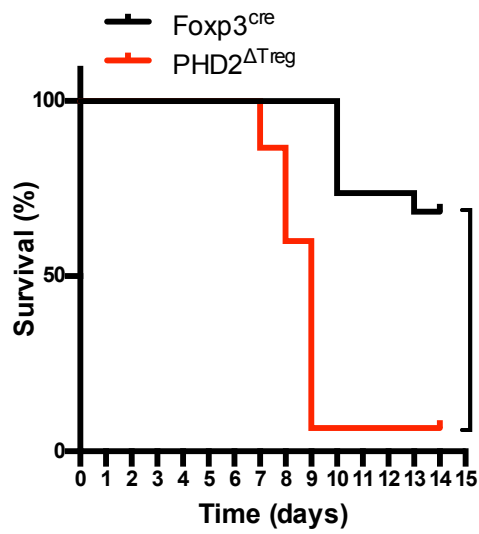

e

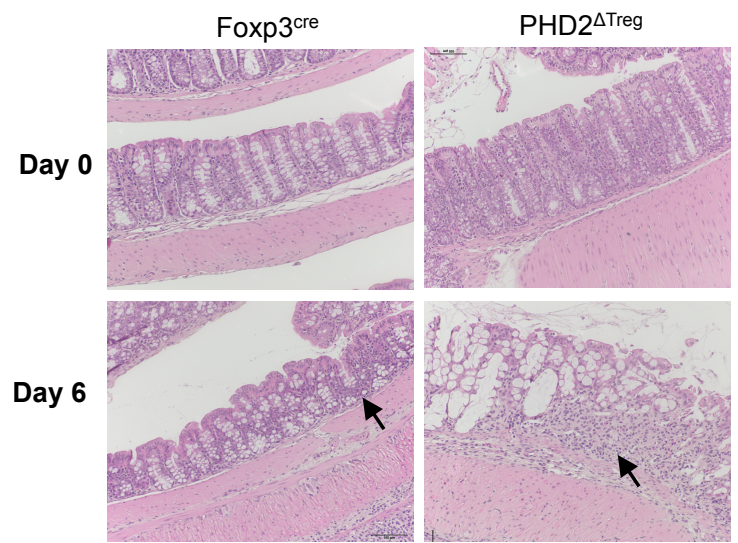

$9 \triangle$ Control

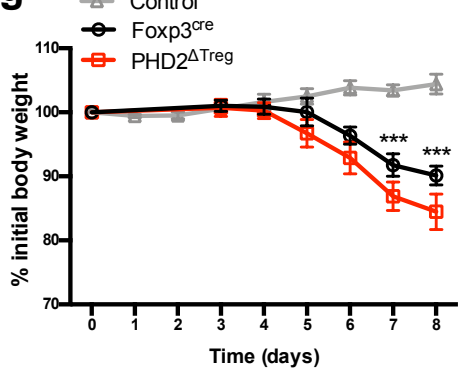

j

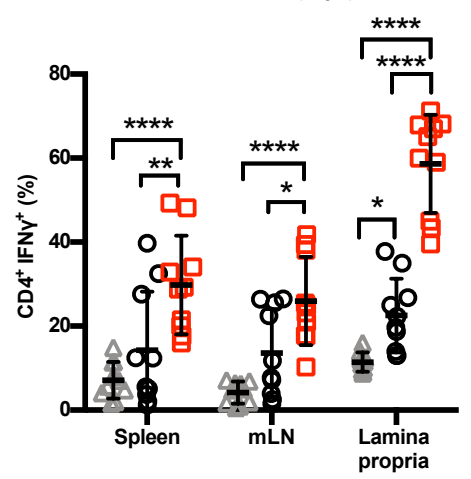

C

- Foxp3 $3^{\text {cre }}$

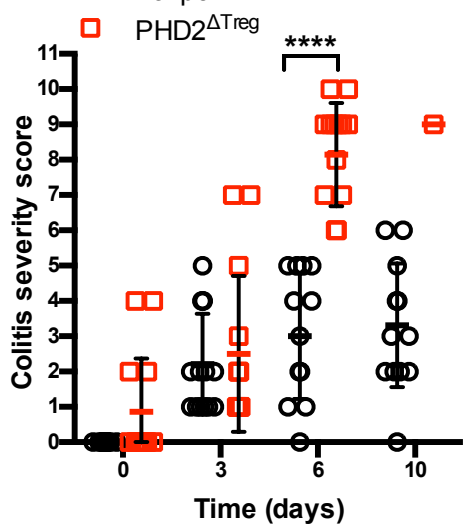

h

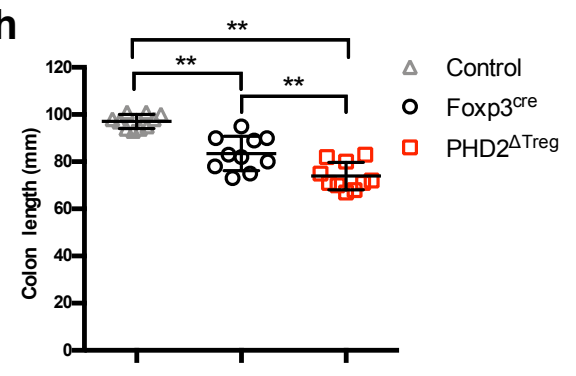

k

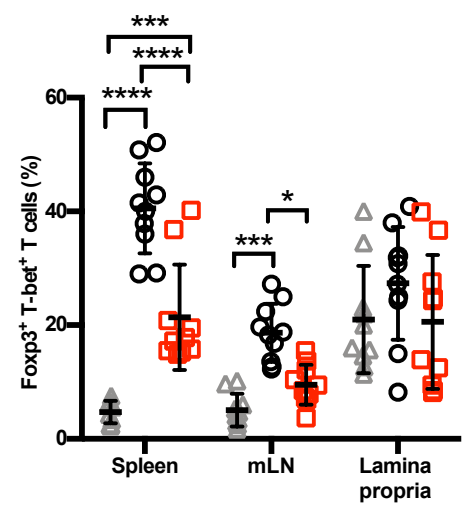

Fig. 4 Increased sensitivity of PHD2 ${ }^{\Delta \text { Treg }}$ mice to DSS-induced colitis and toxoplasmosis. 
a

(which was not certified by peer review) is the author/funder. All rights reserved. No reuse allowed without permission.

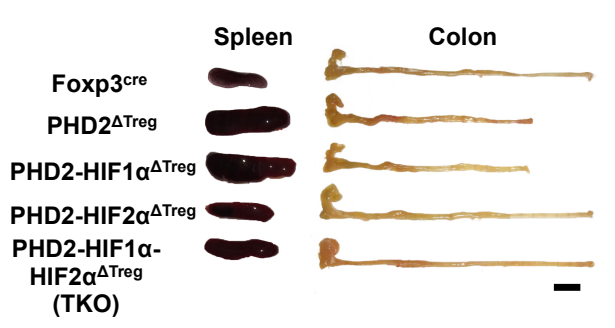

C splenic CD4 ${ }^{+}$

T cells map

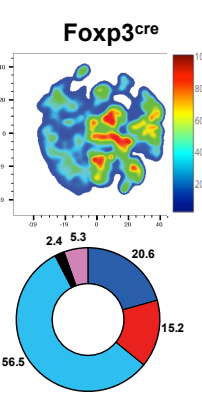

d

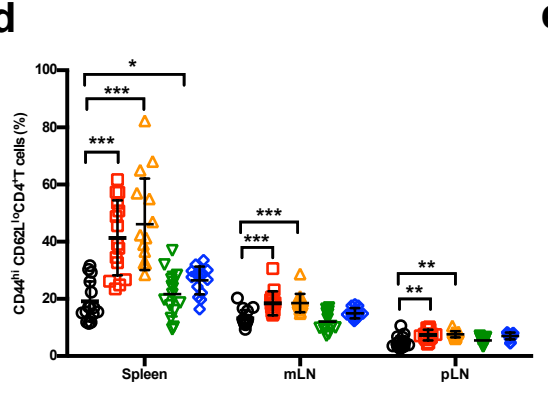

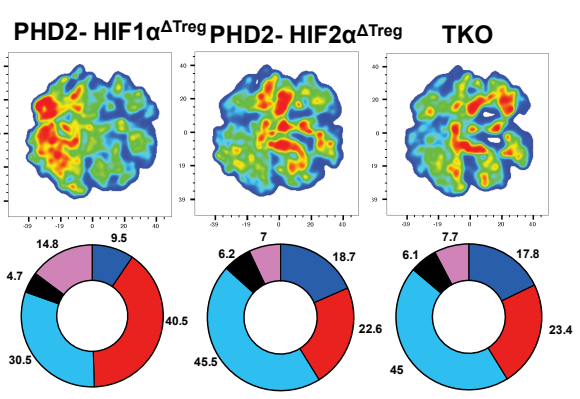

e

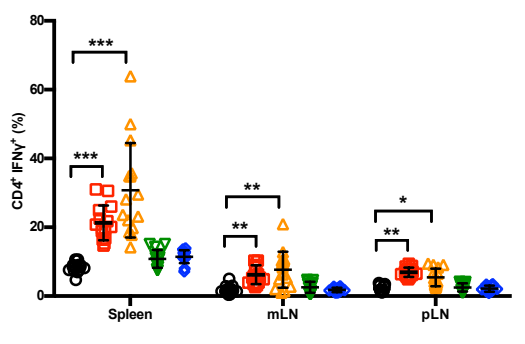

b

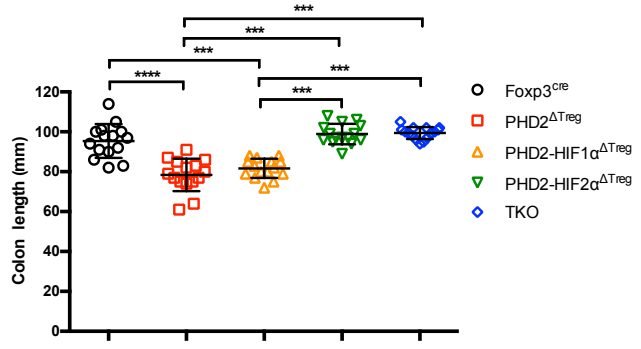

$\mathbf{f}$

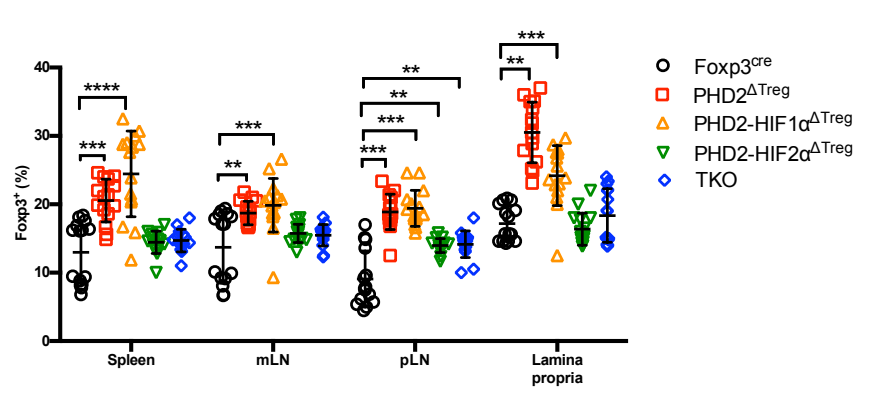

g

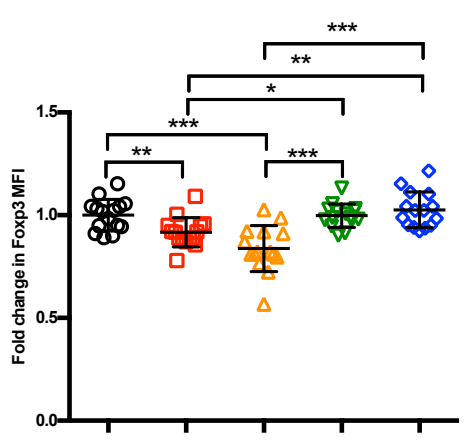

Fig. 5 Concomitant loss of HIF2 $\alpha$ but not HIF1 $\alpha$ expression attenuates the proinflammatory phenotype of PHD2 ${ }^{\Delta T r e g}$ mice. 
a

(which was not certified by peer review) is the author/funder. All rights reserved. No reuse allowed without permission. PHD2-

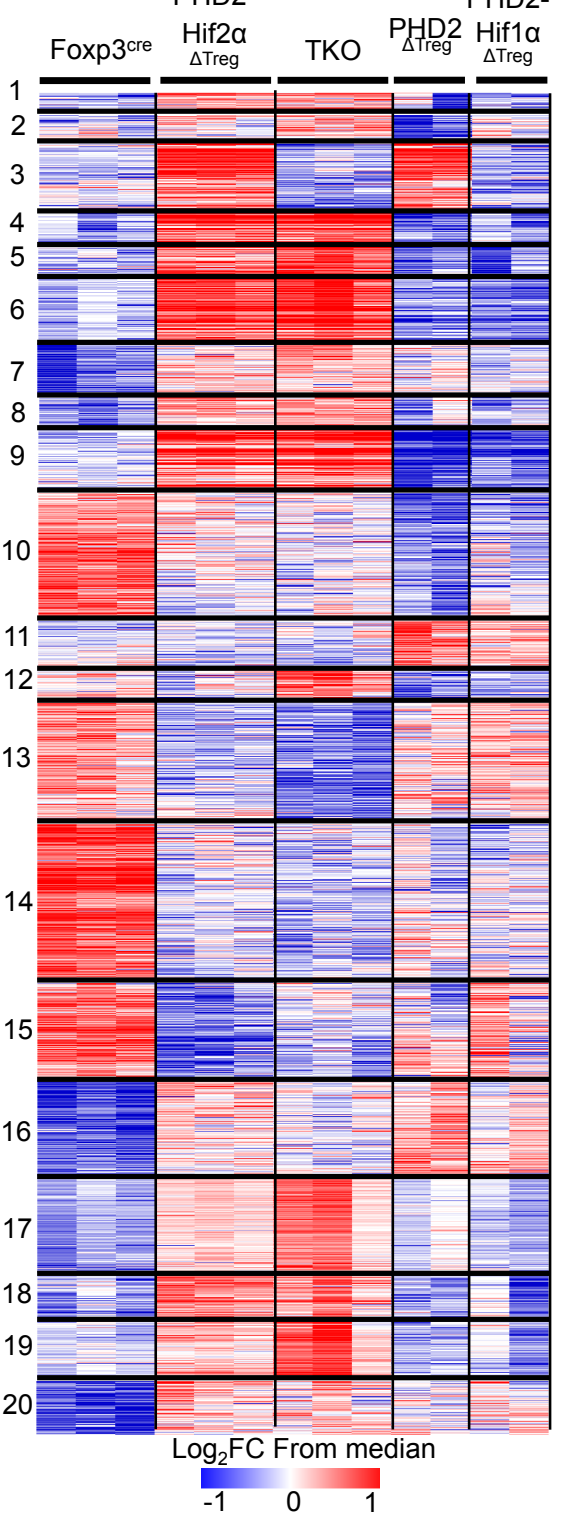

b

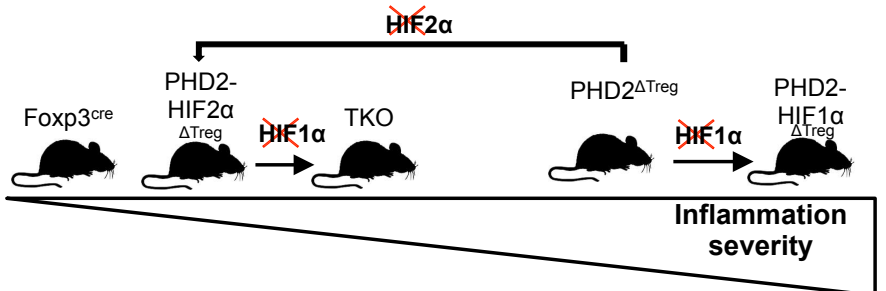

Fig. 6 Anti-inflammatory response, response to chemokines and cell survival pathways represent targets of the PHD2HIF2 $\alpha$ axis in Tregs.
C

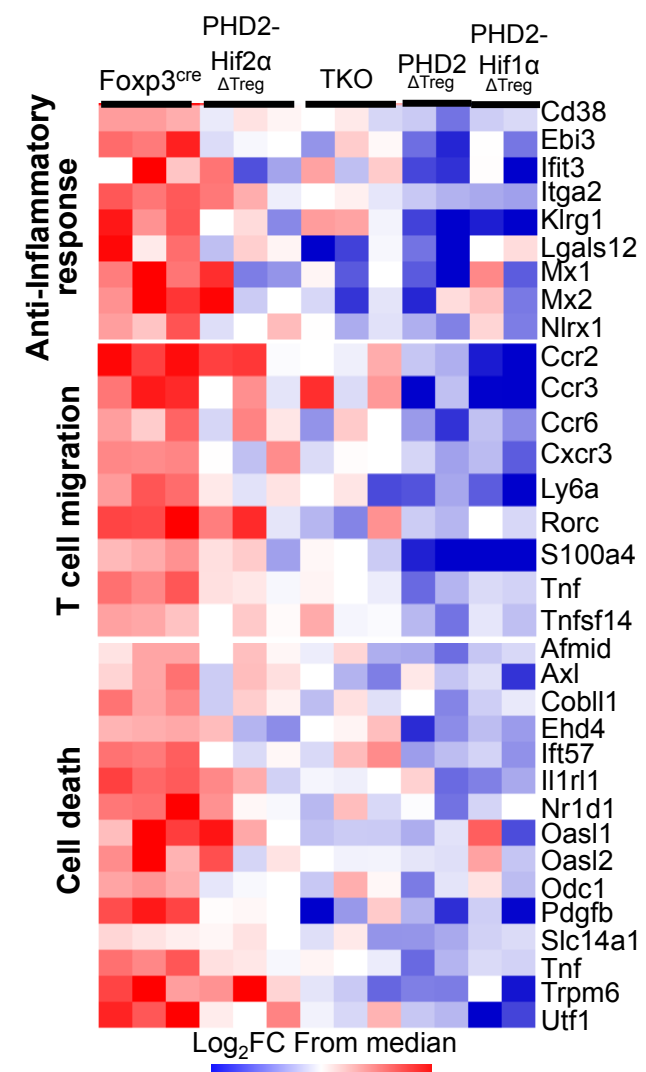

d

e
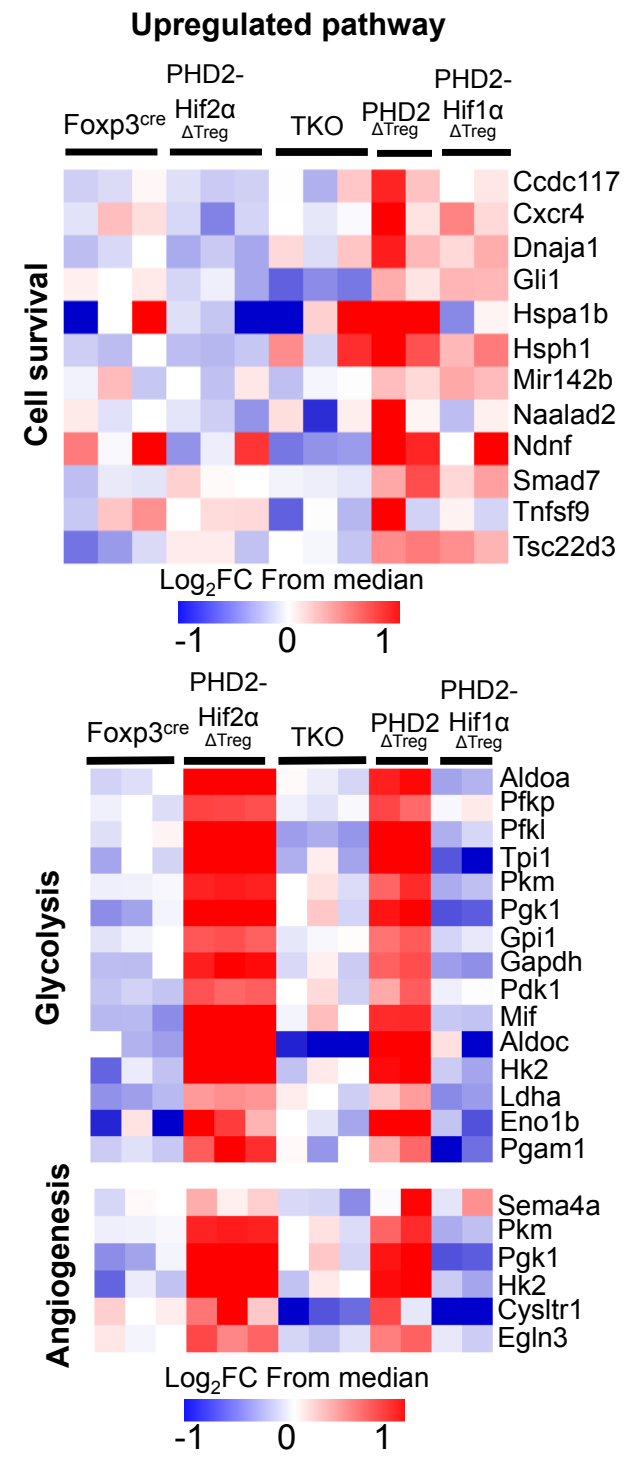
a

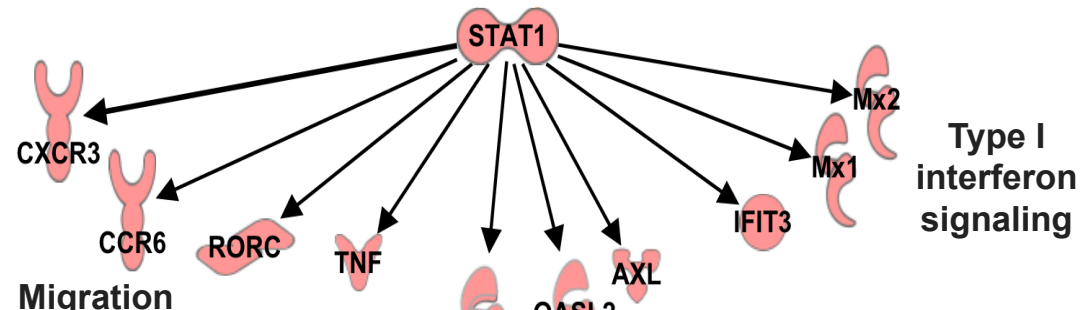

Migration

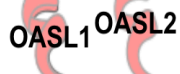

Cell death

b

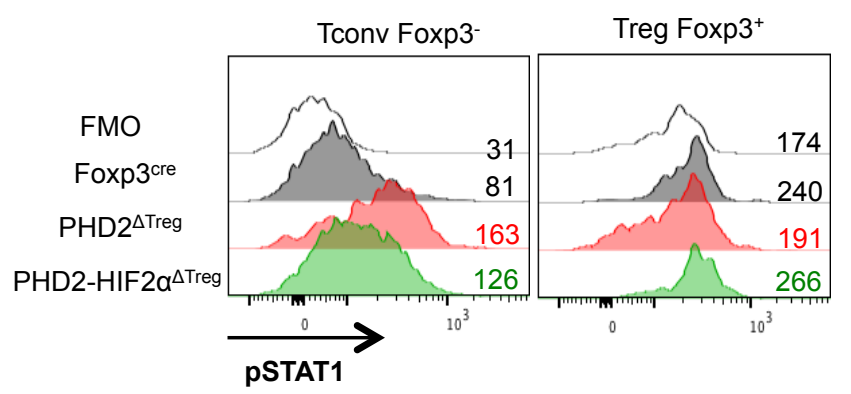

C

$$
\begin{array}{ll}
0 & \text { Foxp3 }^{\text {cre }} \\
\square & \text { PHD2 }^{\Delta T r e g} \\
\nabla & \text { PHD2-HIF2a } \\
&
\end{array}
$$

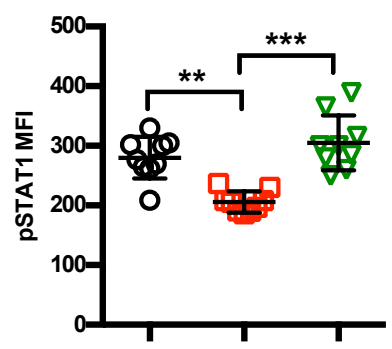

d

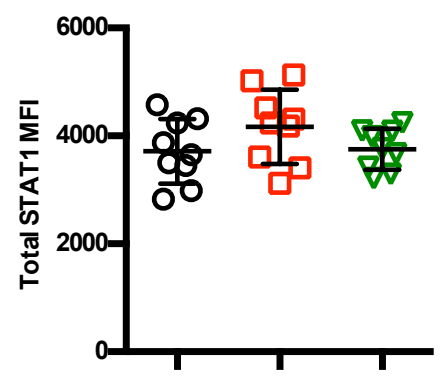

Fig. 7 Identification of STAT1-mediated signaling as a target of the PHD2-HIF2 $\alpha$ axis in Tregs. 
a

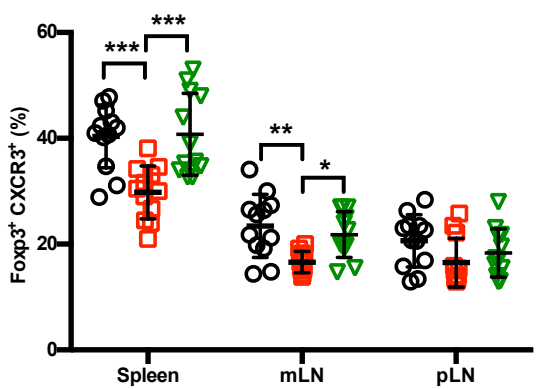

C
- Foxp3 $3^{\text {cre }}$
口 PHD2 ${ }^{\Delta T r e g}$
$\nabla \quad$ PHD2-HIF2 $a^{\Delta \text { Treg }}$

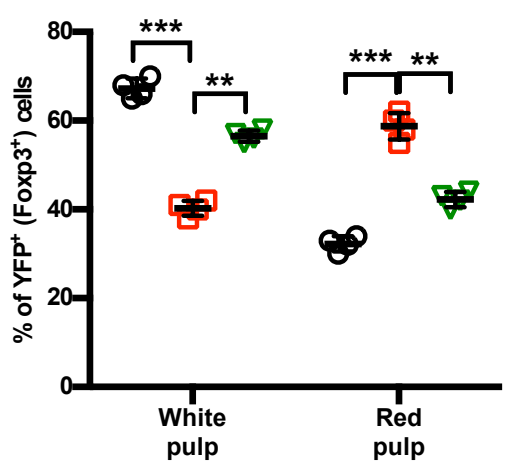

b
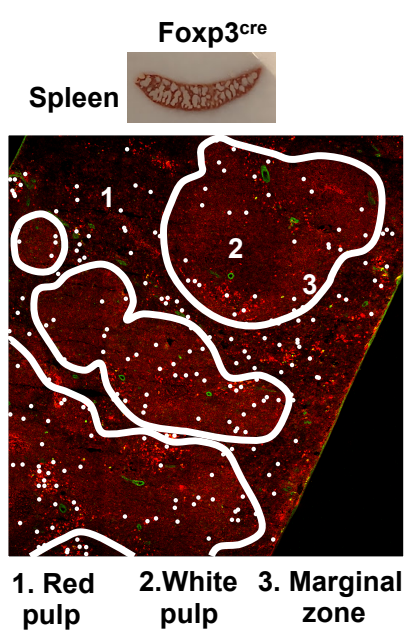

d
PHD2HIF2 $\alpha^{\Delta \text { Treg }}$
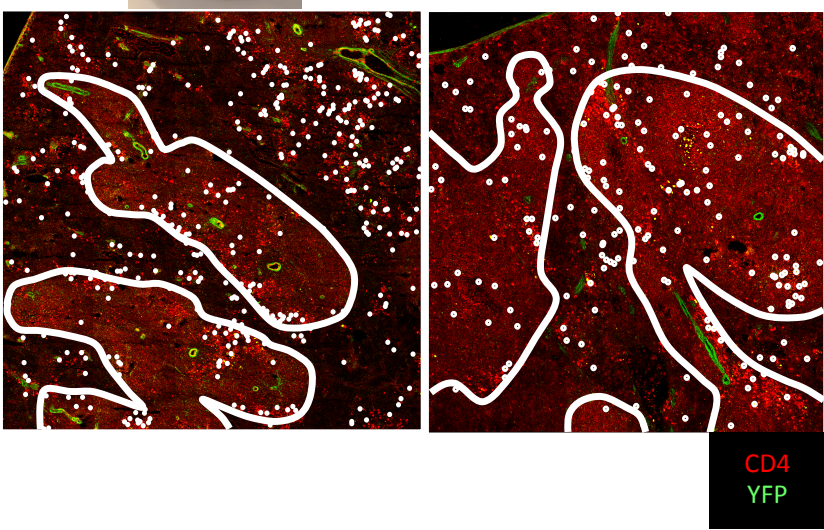

e
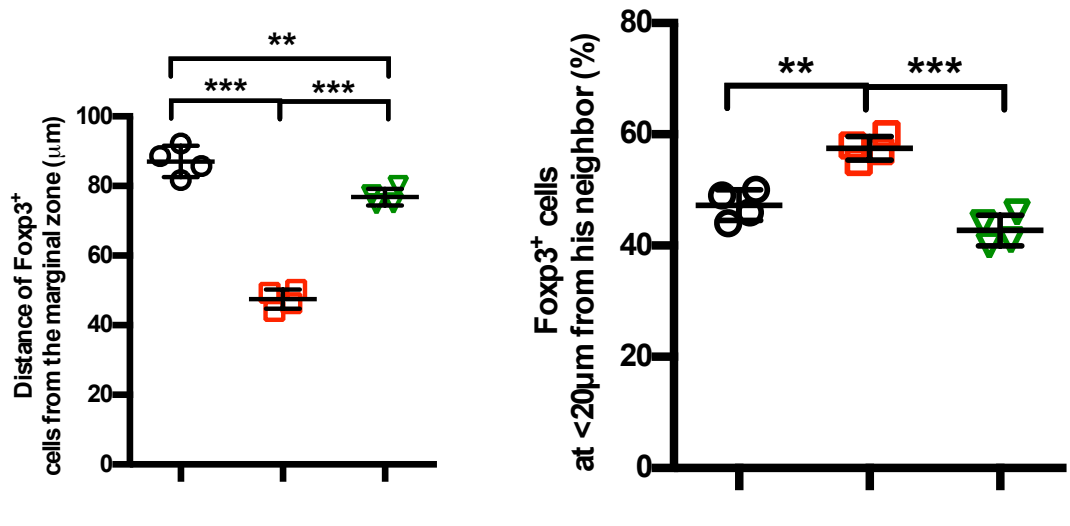

Fig. 8 The PHD2-HIF2 $\alpha$ axis regulates adequate positioning of Tregs in the splenic white pulp. 